\title{
Carbon Nanotubes Synthesis
}

\author{
Eba Medjo Rolant \\ University of Yaoundé I, Faculty of Science, \\ Republic of Cameroon
}

\section{Introduction}

Carbon is the most important element of the periodic classification for human Being. It is known since prehistoric times in form of diamond. Many centuries after, the graphitic form is discovered and used. The other forms completing the traditional forms of carbon, carbynes and amorphous carbons are recently known. The history of modern Carbon Science started more than a hundred years ago, and continues to expand rapidly. In the earlier years of the decade 1890, Hughes and Chambers were studied carbon fibers and filaments. Half century later, reports in hollow of carbon fibers were published, presenting the structures as being composed of many finer threads and twisted, with thickness varying from 10 to 200 nanometers. At that time the demand of improved mechanic properties of carbon materials by space and aerospace industry, led to real progress in production and characterization of carbon based materials.

In the 1970 and 1980 decades, sustained investigations have led to many techniques of synthesis of carbon materials (Baird et al, 1971; Dresselhaus et al, 1988), many methods of characterization and of course an important number of novel carbon allotropes, with a generic name of carbon nanostructures (CNSs). The first discovered carbon nanostructure, is the fullerene in 1985, the second is the carbon nanotube (CNT) in 1991 by Sumio Iijima of the NEC, Japan (Iijima, 1991). Nowadays, there is a plethora of carbon nanostructures. Among these novel nanostructures, carbon nanotube exhibits unique physical and chemical properties. It is promising to revolutionize several fields of fundamental science and contribute as major component of nanotechnology. It can be used in composite materials or in individual functional element of nanodevices such as: hydrogen storage, nanomanipulation, medical usages and nanoporous membranes. Besides the attractive aspects of carbon nanotubes, their synthesis techniques are complex compared to other materials used in different domains of technology and different existing methods of synthesizing. These methods lead to CNTs with sometimes an important quantity of impurities incorporated, encapsulated or adsorbed, whose amount and types depend on techniques and parameters of synthesis.

This chapter provides information in the versatility of carbon element, allowing him to form more than $50 \%$ of known chemical compounds. There is a considerable amount of literature on the study of carbon nanotubes grown by varieties of synthesis techniques. The procedures most commonly used include: arc discharge, laser ablation and catalytic decomposition of carbon-containing compounds over catalyst: the so-called chemical vapour deposition (CVD) techniques on a flat substrate (Dresselhaus \& Endo, 2001). This 
last method has attracted much attention due to the fact that it appears to be a promising technique for the production process at relatively low cost and mass quantities. It has become the most popular technique of CNTs synthesis and can lead to a controlled growth of the CNTs by varying operating parameters such as gas mixture, temperature, pressure and catalyst.

\section{Carbon hybridization}

In the nature, there are some pure materials that have striking different properties even made of the same atoms, carbon is one of them. Found in abundance in the universe, in the sun, in the stars, the comets and in the atmosphere, carbon is also the basic component of all organic matter. It is found everywhere from the graphite in a pencil, to the soot in a chimney. Two very popular materials: graphite and diamond could not be more different. Both are made of carbon atoms linked through strong bonds. Natural carbon exists in two isotopic forms, ${ }^{12} \mathrm{C}$ and ${ }^{13} \mathrm{C}$. The nucleus of the abundant isotope of carbon ${ }^{12} \mathrm{C}$ is composed of six protons and six neutrons. Neutral carbon atom is tetravalent and has totally six electrons with four of them occupying the outer orbit. Therefore, the electronic configuration of the carbon atom at fundamental state is $1 s^{2} 2 s^{2} 2 p_{x}^{1} 2 p_{y}^{1} 2 p_{z}^{0}$ and does not explain several bonds of carbon structures. According to organic chemistry, one of the two $2 s$ electrons is promoted to $2 p_{z}$ orbital. So the electronic wave functions for the four weakly bound electrons can mix each others, thereby changing the occupation of the $2 s$ and $2 p$ orbitals, since the energy difference between the lower $2 s$ and the upper $2 p$ levels is low compared to the binding energy in the chemical bonds. This mixing of atomic orbitals is called hybridization. In carbon atom, three possible hybridizations occur denoted $s p, s p^{2}$ and $s p^{3}$.

In the $s p$ hybridization, there is linear combination of $2 s$ orbital and one of the three $2 p$ orbitals, $2 p_{x}$ for example. From these two atomic orbitals, two equivalent orbitals are formed, called hybridized orbitals and, are expressed by linear combinations of $|2 s\rangle$ and $\left|2 p_{x}\right\rangle$ as illustrated in Fig. 1, and denoted $\left|s p_{a}\right\rangle$ and $\left|s p_{b}\right\rangle$.

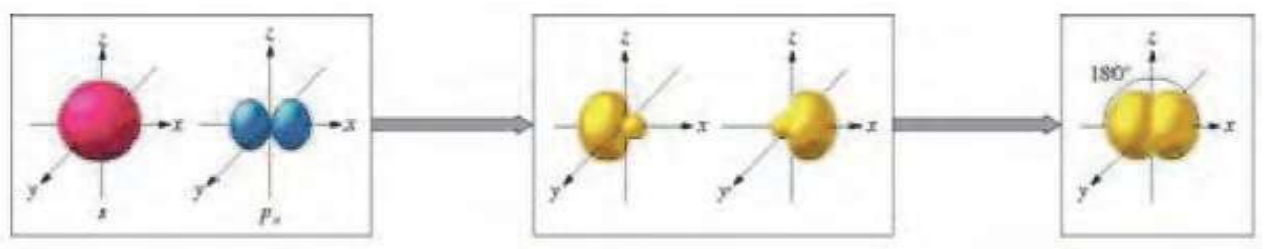

Fig. 1. Sp hybridization and form of resulting structure orbitals of carbon atom

In the $s p^{2}$ hybridization, three atomic orbitals are involved. The $2 s$ and two of $2 p$ orbitals, for example $2 p_{x}$ and $2 p_{y}$, are mixed. The three obtained hybridized orbitals are in the same plane and form three $\sigma$ bonds in molecules. The resulting structure is planar. The hybrid atomic orbitals obtained have large amplitude in the directions of the three nearest neighbour atoms. These three directions are denoted by a trigonal bonding, as shown in Fig. 2 . 


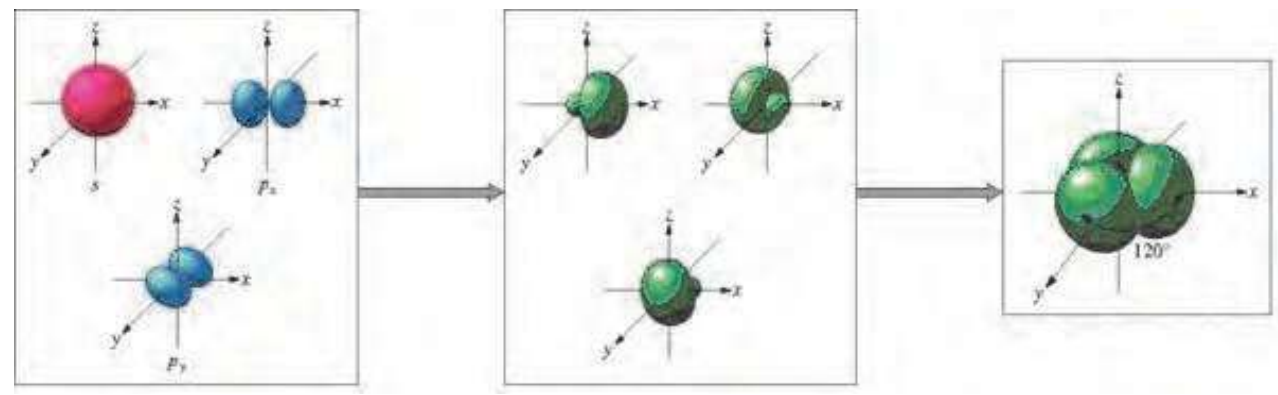

Fig. 2. Illustration of $s p^{2}$ hybridization and resulting structure.

The carbon atom provides a $s p^{3}$ hybridization through its tetragonal bonding to four nearest neighbour atoms which have the maximum spatial magnitude from each other as graphically presented in Fig. 3. The four directions of tetrahedral bonds from the carbon can be selected as $(1,1,1),(-1,-1,1),(-1,1,-1)$ and $(1,-1,1)$. In order to make elongated wave functions to these directions, the $2 s$ orbital and the three $2 p$ orbitals are mixed, forming a $s p^{3}$ hybridization.

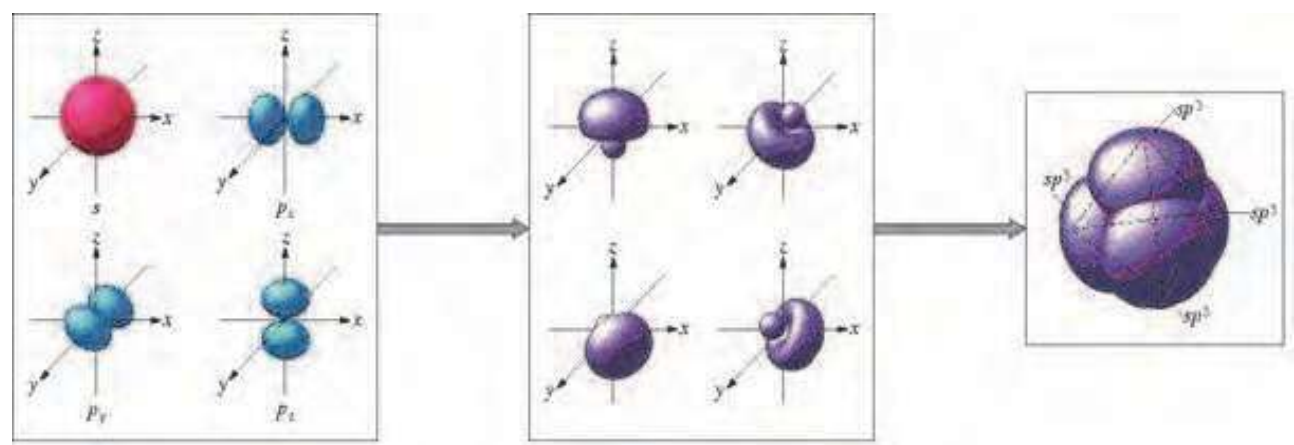

Fig. 3. Illustration of $s p^{3}$ hybridization and resulting structure.

\section{Carbon allotropes}

Carbon is known as the king of chemical elements owing to its versatility and diversity in all fields. It is known to form nearly ten millions different compounds. Carbon holds the sixth place in the list of abundance in Universe. First of all, life is based on carbon. Secondly, the several allotropic forms of carbon have very peculiar and interesting properties. It is known in form of diamond earlier and has been prized for centuries as a gemstone of exceptional beauty, brilliance and lustre. In the form of graphite, it is used since the middle of the $15^{\text {th }}$ century as a writing material. A graphitic basal plane or a graphene layer can be defined as an hexagonal network of covalently bonded carbon atoms or a single two-dimensional (2D) layer of three-dimensional (3D) graphite. The compounds of pure carbon have two traditional allotropes known since thousands of years or centuries. They are: diamond, a hard and colourless solid of carbon atoms $s p^{3}$ 
hybridized, and graphite soft and black solid with $s p^{2}$ atoms hybridization. The other pure carbon allotropes of this group are the carbynes and the amorphous carbons. The forms discovered from 1985 to now are carbon nanostructures.

\subsection{Traditional forms of carbon}

\subsubsection{Diamond}

Diamond always comes on top when compared to any other material. It has been the hardest known substance till the recent two decades and is inert to chemical corrosion and can withstand compressive forces and radiations. Diamond is transparent to visible light, $\mathrm{X}$ rays, ultraviolet radiations and much of the infrared spectrum. It conducts heat better than any other material. Fig. 4 bellow shows an image of diamond by electron microscope.

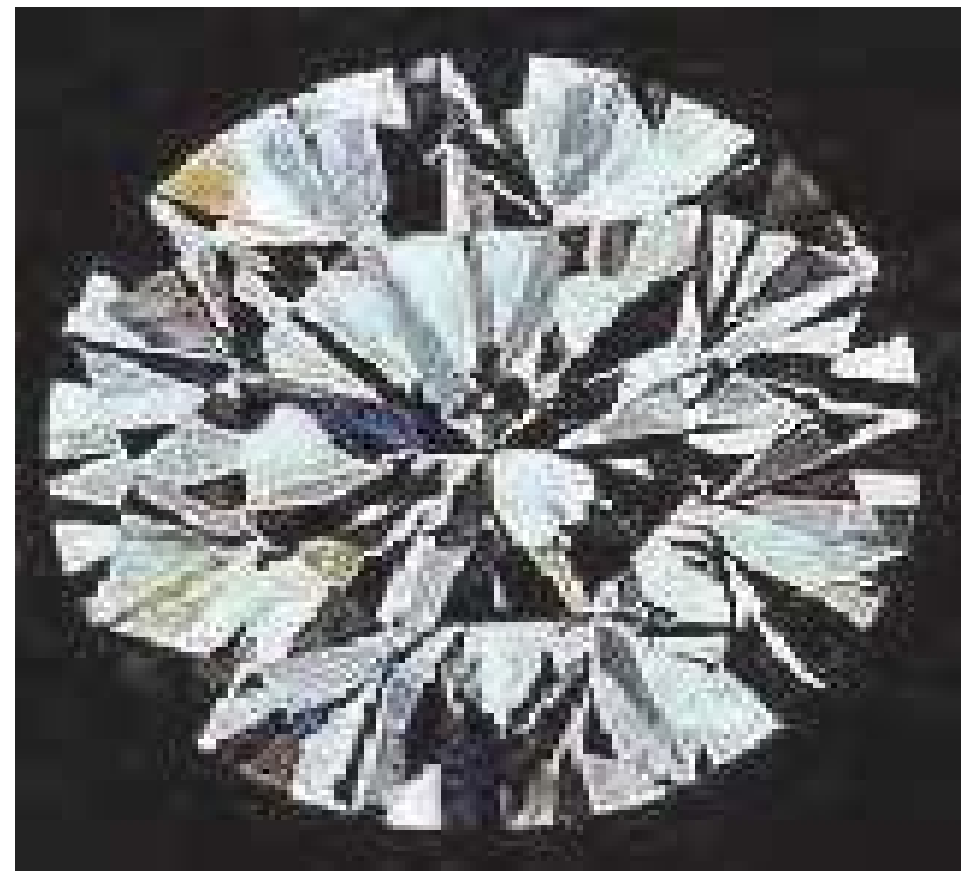

Fig. 4. Diamond's electron micrograph

At room temperature, it has the highest thermal conductivity than any solid (Dolmatov, 2001). These impeccable qualities arise from the elemental nature of carbon and its bonding structure. In diamond, each carbon atom is covalently single bonded to four other carbon atoms by strong $\sigma$ bonds. Diamond is now produced by CVD. In this low-pressure technique, artificially produced diamonds have less impurities and crystalline defects, and are affordable and accessible for use in science and industry. Naturally occurring diamond is almost always found in the crystalline form with a purely cubic orientation of $s p^{3}$ bonded carbon atoms as illustrated in Fig. 5, while synthetic diamond is a randomly mixture of cubic and hexagonal lattices. In special conditions, carbon crystallizes in lonsdaleite, which is a form similar to diamond but with hexagonal structure. 


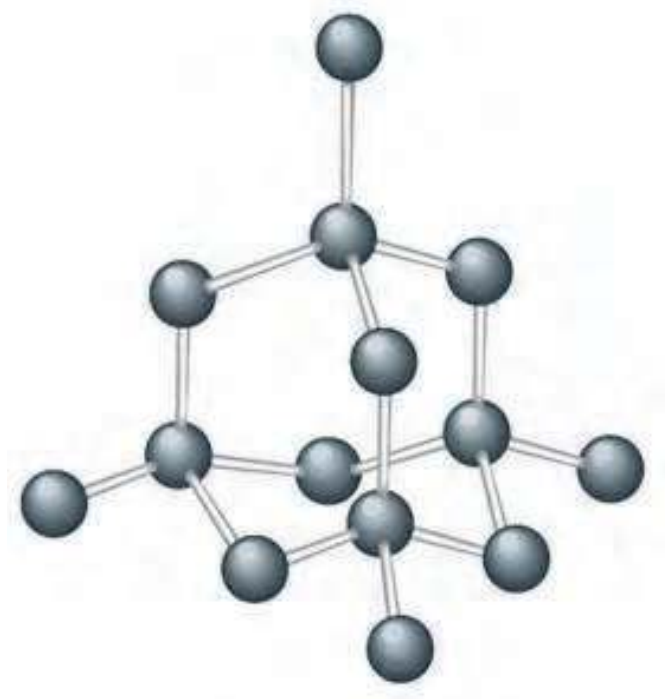

Fig. 5. Cubic structure of natural diamond

\subsubsection{Graphite}

Graphite is a highly anisotropic solid. Structurally, its interplanar spacing (3.35 $\AA$ ) is quite large compared to the in plane interatomic spacing (1.42 $\AA$ ). Physically, its stiffness along the plane is quite large because of strong $\sigma$ bonds and in the perpendicular direction; it is weak because of the Van der Waal's forces. The planes can be cleaved easily making graphite quite a soft material. Also electronically it is anisotropic, because of $\pi$ and $\pi^{*}$ bands overlap. This has metallic conductivity along the plane and semi-conducting perpendicular to the plane.

The anisotropy in conductivity is about $10^{3}$. The $s p^{2}$ hybridization forms a planar structure. It is made of strong fibers composed of series of stacked parallel layers as shown in Fig. 6. It is black and lustrous, optically opaque, unaffected by weathering. Its greasy frictionresistant properties allow for applications in lubricating oils and greases, dry-film lubricants, batteries, conductive coatings, electrical brushes, carbon additives and paints. The graphite is the most stable form of pure carbon structures, stacked with an offset between neighbouring sheets of $1 / 2$ of a lattice constant in an A-B-A-B layering. The sheets are separated by a larger distance $(3.35 \ddot{A})$ and interact weakly with a mixture of covalent and Van der Waals bonding that gives graphite strong lubricating properties, as pieces of graphite planes can glide along each other almost frictionless. The weak bonding of graphite in the perpendicular direction to the planes (Pierson, 1993) makes the synthesis of monocrystalline graphite almost impossible since the planes can shift with respect to each other, and they can also rotate. This gives the so-called turbostatic graphite, which has very crystalline planes, but with orientational disorder between them. The best commercial graphite is highly-oriented pyrolitic graphite (HOPG) which has a reduced disorder, but still remains polycrystalline. The theoretical interest in being able to play with true monocrystals of graphite is immense (Novoselov et al., 2004; Bunch et al., 2005). 


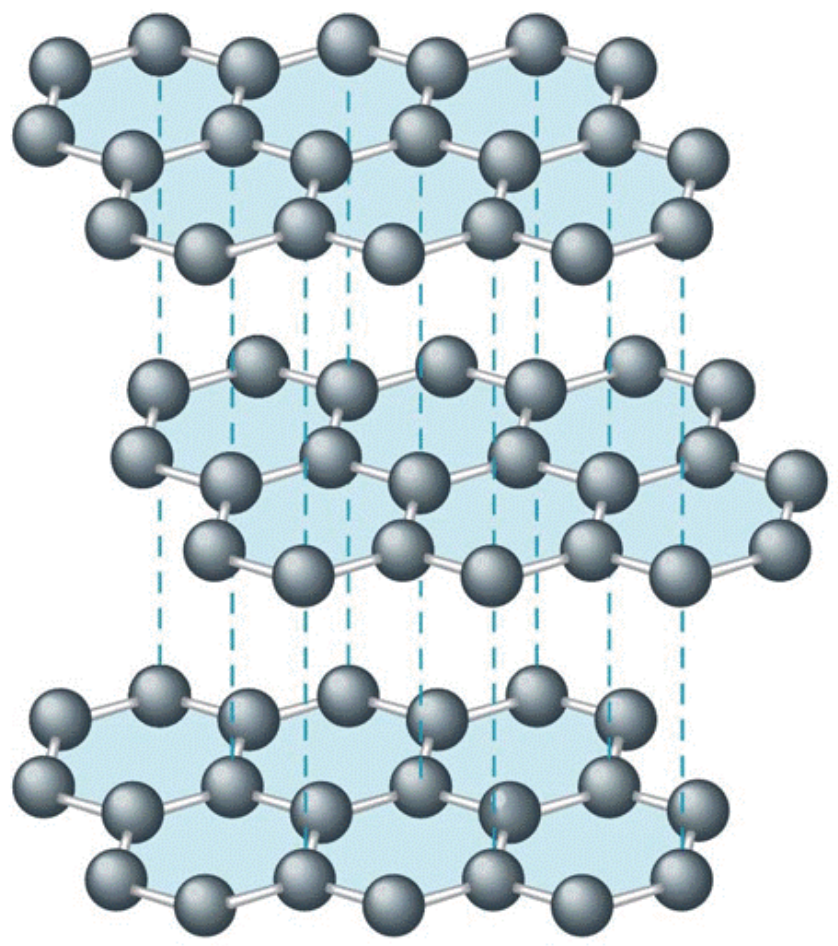

Fig. 6. Hexagonal structure of Graphite in an A-B-A-B layering

\subsubsection{Carbynes}

Linear chains of carbons which have $s p$ bonding or carbynes have been subject to intensive research for many years (Heimann et al., 1999). These carbon structures are stable at high temperature and pressure. $s p$ carbon chains can present:

a. alternating single and triple bonds; the polyyne, with the following chemical structure

$$
[-C \equiv C-C \equiv C-] n
$$

b. only double bonds; the polycumulene, chemically structured as follow

$$
[=C=C=C=C=] n \text { with } n>10 \text { (Heimann et al., 1983). }
$$

Theoretical calculations suggest that polycumulenes are less stable than polyynes (Heimann et al., 1983). Both species are characterized by an extremely high reactivity with oxygen, hydrogen and a strong tendency to interchain cross linking (Heimann et al., 1999), thus rendering the direct observation of a pure carbyne-assembled solid is still a major challenge. Carbynes are silver-white coloured and are found in meteoritic carbon deposits, where they are mixed with graphite particles. Synthetic carbynes have also been prepared by sublimation of pyrolytic graphite. They are formed during very rapid solidification of liquid 
carbon, near the surface of the solidified droplets formed upon solidification. In the solid form, carbynes have a hardness intermediate between diamond and graphite.

\subsubsection{Amorphous carbons}

The amorphous carbons or free reactive carbons are carbon allotropes that do not have any crystalline structure. Scientific research on amorphous forms of carbon has found importance in recent decades owing to its rich underlying physics and tremendous applications. The widely known forms of amorphous carbons are: black of carbon, carbon fibers, porous carbon, glassy carbon, diamond like carbon (DLC) and pyrocarbon. Amorphous carbons $(a-C)$ and diamond-like carbon $(t a-C$ or DLC) solids are characterized by a large varieties of types and properties that stem from combinations of principally two hybridized forms of carbon $s p^{2}$ and $s p^{3}$. The properties of amorphous carbon films depend on the parameters used during deposition. One of the most common ways to characterize amorphous carbons is through the ratio of $s p^{2}$ to $s p^{3}$ hybrid bonds present in the material. Amorphous carbons are usually applied as coatings to other materials.

\subsection{Carbon nanostructures}

\subsubsection{Fullerenes}

Fullerenes are molecules consisting of carbon sheets of hybridized $s p^{2}$, forming a closed sphere structure. These spherical structures are built up out of hexagons and pentagons. The fullerene's formation is based on the introduction of pentagonal, heptagonal or other kind of "defect" rings between the hexagonal rings of the graphene sheets, which favour a higher curvature of the sheet. Each fullerene, by definition, consists of 12 pentagons (Fig. 7), since a sphere containing $n$ hexagons cannot be closed otherwise according to Euler's theorem.
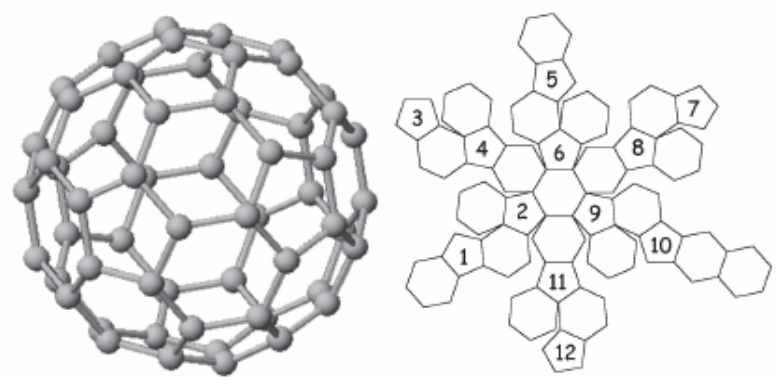

Fig. 7. Fullerene $C_{60}$. The necessary twelve pentagons to fulfil Euler theorem are numbered

$C_{60}$ is made of 20 hexagons and 12 pentagons. Each carbon atom is at the intersection of two hexagons and one pentagon. When compared to planar graphene, the introduction of a pentagon gives a positive curvature to the surface. Euler's rules dictate that to create a closed surface, we need a total "curvature charge" of 12 (Hamon et al., 2001). As a result, all the simple fullerenes will have the same number of pentagons, 12, and differ only by the number of hexagons inserted between them. The extreme small fullerene $C_{20}$ is too curved to be stable, as are the other intermediate fullerenes before $C_{60} \cdot C_{60}$ defines the "isolated 
pentagon" rule: all pentagons in a stable fullerene must be surrounded by hexagons. The smallest sphere fulfilling these conditions is $C_{60}$.

This molecule, consisting of sixty carbon atoms, forms a perfect "soccer ball" structure. Due to its similarity to the domed houses dreamed up by the architect Buckminster-Fuller, the molecules were named Buckminster fullerenes or fullerenes in short. New and larger shapes were discovered as shown in Fig. 8, with 70, 76, 82, 84... carbon atoms (Kroto, 1987), and even more. Sometimes fullerenes fit together concentrically forming bucky onions (Mordkovich et al., 2002).

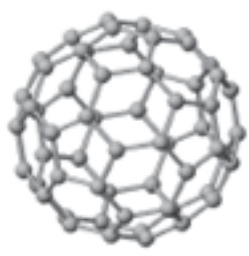

$\mathrm{C} 60$

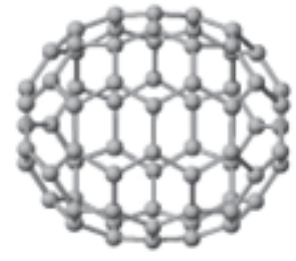

$\mathrm{C} 70$

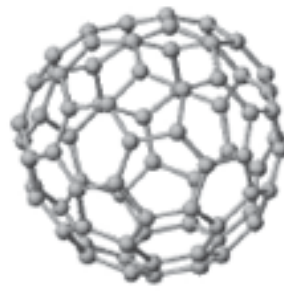

C76

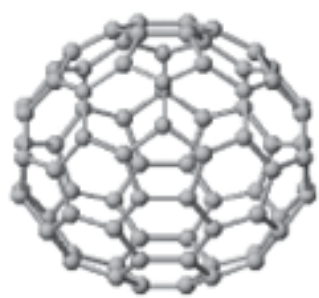

C78

Fig. 8. Some fullerenes.

\subsubsection{Carbon nanofibers}

Carbon nanofibers (CNFs) are conical structures that have diameters varying from a few to hundreds of nanometers and lengths ranging from less than a micron to millimeters. The internal structure of carbon nanofibers varies and is comprised of different arrangements of modified graphene sheets. In general, CNFs have a structure which is a mixture of aligned graphene planes as well as a bamboolike or cup-stacked structure, previously ascribed to herringbonelike structure, in other words a nanofiber consists of stacked curved graphite layers that form cones or "cups" (Krishnan et al., 1997; Melechko et al., 2005). Currently there is no strict classification of nanofiber structures. The main distinguishing characteristic of nanofibers from nanotubes is the stacking of graphene sheets of varying shapes. Defining $\alpha$ as an angle between the fiber axis and the graphene sheet near the sidewall surface, nanofiber with $\alpha=0$ is a special case in which, one or more graphene layers form cylinders that run the full length of the nanostructure. This arrangement, with its closed and semiinfinite surface results in extraordinary properties that made this type of nanofiber known to the world as a carbon nanotube.

\subsubsection{Carbon nanotubes}

In 1991, Sumio Iijima discovered (Iijima, 1991) yet another fascinating form of carbon, in the soot recovered from the discharge of an electrical arc between graphite electrodes. He found ultra-thin tubules of carbon: the carbon nanotube. Since this period, countless papers on carbon nanotubes, their properties, and applications have appeared and generated great interest for future applications based on their field emission and electronic transport properties, their high mechanical strength and chemical properties (Hueczko, 2002; Meyyappan et al., 2003). Carbon nanotubes can be described as cylindrically shaped molecules formed of rolled up single or multilayer sheets of graphitic planes presented on 
Fig. 9. In other words, it can be considered as an infinite strip cut out of a graphene sheet and rolled up along the direction perpendicular to the strip. Geometrically, CNTs have one of the highest aspect ratio of any object in nature. Their length can exceed several millimeters for diameters around ten nanometers. The characteristics of a carbon nanotube, and the position of every atom in it, can be determined by just two integers. All what is needed, it is the circumference of the tube, which joins the two equivalent atoms along the circumference. These will be superimposed once the strip is rolled up.

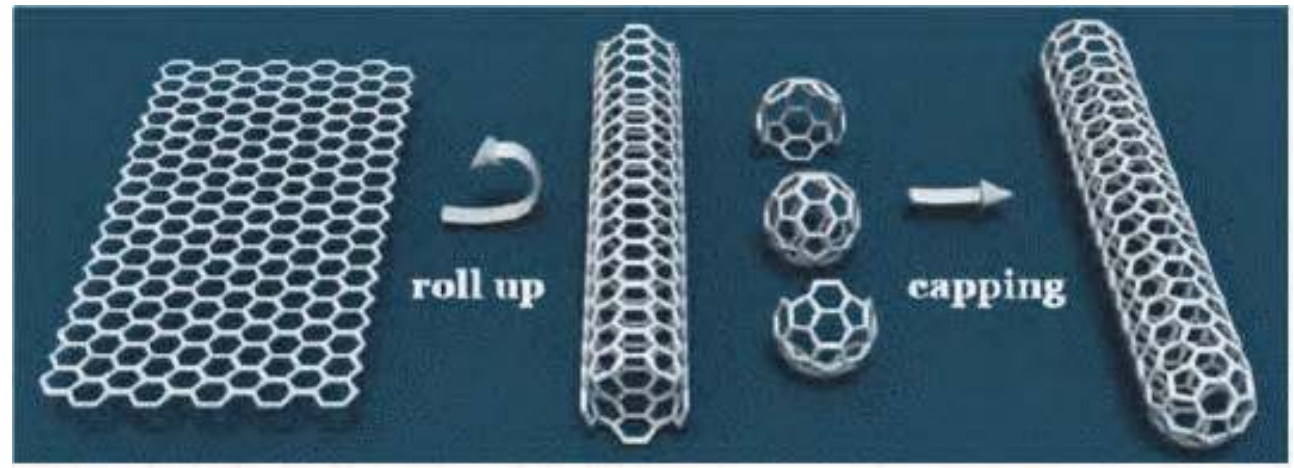

Fig. 9. SWCNT capped with half of a $C_{60}$ fullerene molecule.

The vector between the two graphene's atoms $\vec{C}_{h}$, called the chiral vector is part of the 2 dimension (2D) crystalline lattice, and denoted by the two integer indices, $\mathrm{n}$ and $\mathrm{m}$ called Hamada integers (Hamada et al., 1992). If $\vec{u}$ and $\vec{v}$ are the lattice vectors of a 2D graphene plane as schemed in Fig. 9, the chiral or helicity vector is expressed by:

$$
\vec{C}_{h}=n \vec{u}+m \vec{v}
$$

The circumference of the tube $\mathrm{L}$ is the length of $\vec{C}_{h}$ and is expressed by:

$$
L=a_{C-C} \sqrt{3\left(n^{2}+m^{2}+n m\right)}
$$

Experimentally, for an inter-atomic distance $a_{C-C}=1,44 \AA$, the diameter of the tube is

$$
D_{t}=\frac{L}{\pi}=\frac{a_{C-C} \sqrt{3\left(n^{2}+m^{2}+n m\right)}}{\pi}
$$

Here $a$ is the in-plane lattice constant, equal to about $a=2,49 \AA$

Various types of carbon nanotubes are characterized by their respective chiral angles $\theta$

$$
\left\{\begin{array}{l}
\sin \theta=\frac{\sqrt{3 m}}{2 \sqrt{n^{2}+m^{2}+n m}} \\
\cos \theta=\frac{2 n+m}{2 \sqrt{n^{2}+m^{2}+n m}}
\end{array}\right.
$$


$\theta$ is so that $0 \leq \theta \leq 30^{\circ}$.

The determination of $(n, m)$ and other physical properties, in order to establish correlations and use theoretical models, is still a hot topic of research. CNTs are much smaller than most of the vapour-grown carbon fibers (VGCFs) that have been studied for many years, but the smallest VGCFs often closely resemble to CNTs in their external morphology. However, CNTs are clearly distinguished from VGCFs by the fact that a perfect CNT is a single crystalline grain, and even a defective CNT is a single crystal in cross section, except where the defect occurs. The simplest termination is a hemispherical cap formed by a half of fullerene. General rules have described the topology of the termination as a function of the Hamada indices $(n, m)$. The prototypical example is shown in Fig. 9, but CNTs can also be open ended. According to the integers $n$ and $m$, CNTs can be metallic, if $n-m$ is a multiple of 3 , and semiconductor otherwise.

The exceptional low-dimensionality and symmetry of carbon nanotubes are at the origin of their spectacular physical properties governed by quantum effects. A carbon nanotube can be one of three types, depending on the orientation along which the graphitic planes are folded. When the value of $\theta$ is, respectively, $0^{\circ}, 30^{\circ}$ or takes any intermediary value, a nanotube is respectively called zigzag, armchair or chiral as illustrated in Fig. 10 bellow.

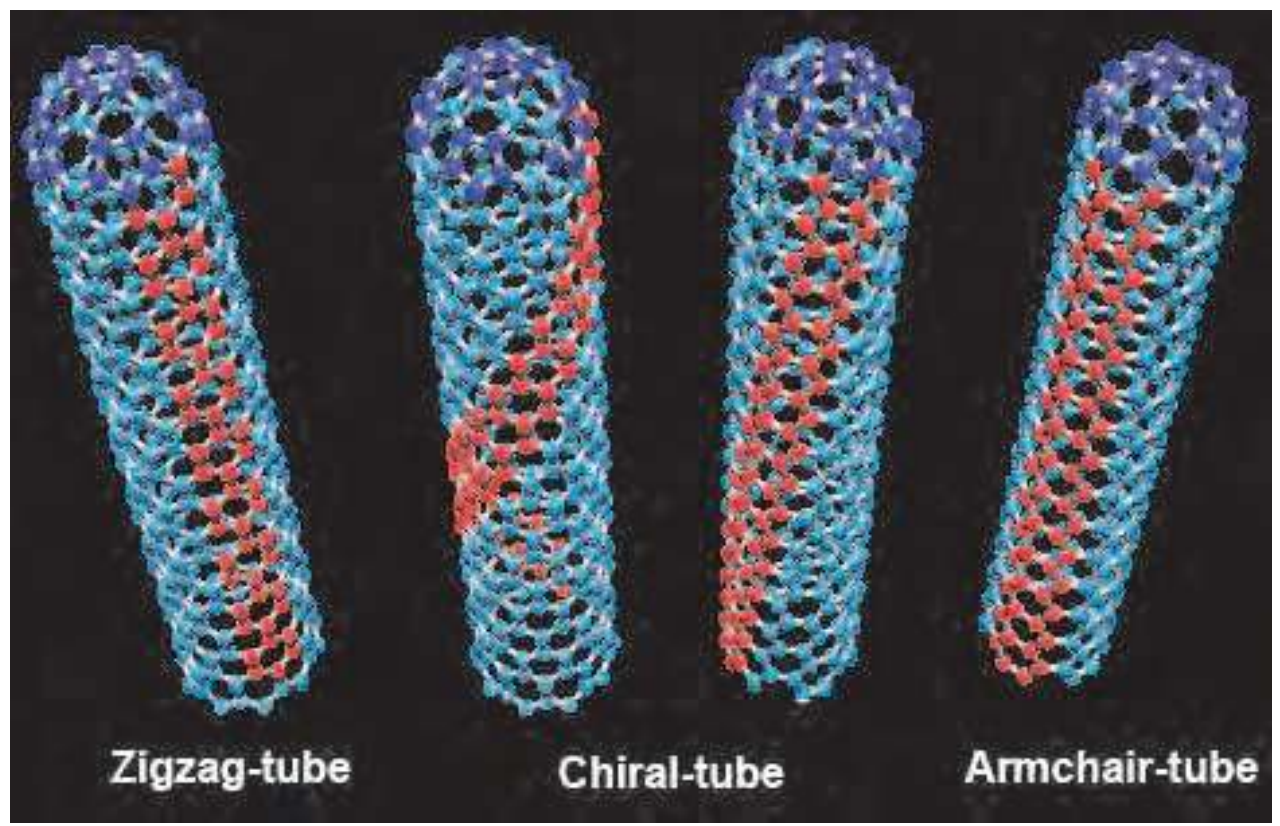

Fig. 10. Possible vectors specified by the pairs of integers $(n, m)$ for general carbon nanotubes including armchair, zigzag, and chiral.

Two types of CNTs can be distinguished; single walled carbon nanotubes (SWCNTs) and multi-walled carbon nanotubes (MWCNTs) as seen in Fig. 11. They can have different properties one another.

Typical outer diameters are approximately 1-6 $\mathrm{nm}$ for SWCNT and 6-100 nm for MWCNT. MWCNT can be rolled up with their graphene layers concentric or spiral. 


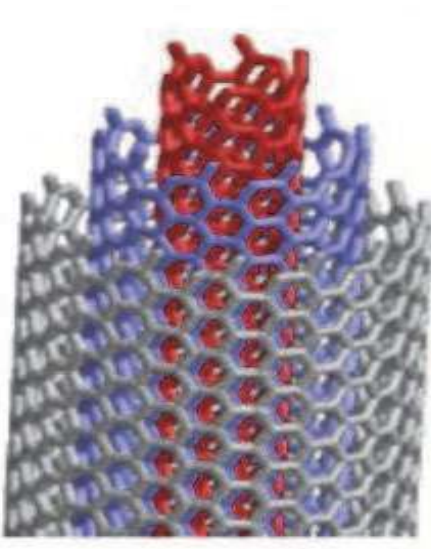

(a)

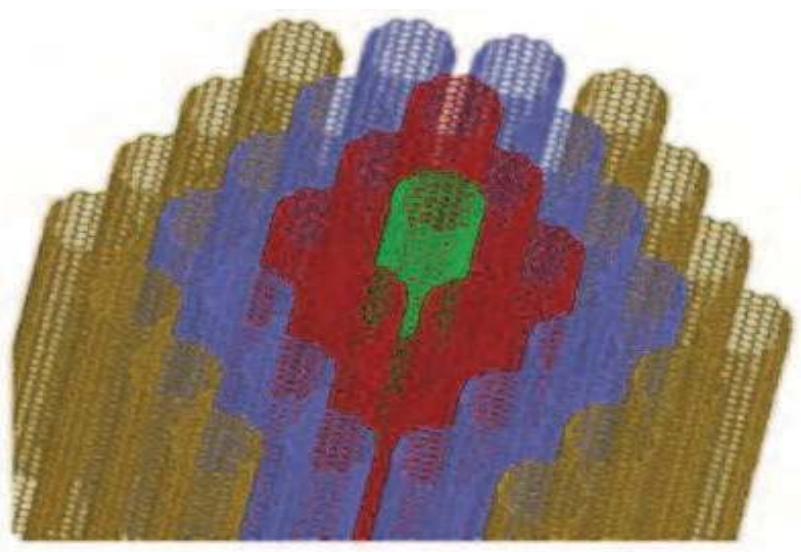

(b)

Fig. 11. Different kinds of CNTs with regard to the number of walls: (a) SWCNT and (b) MWCNT.

\subsubsection{Other carbon nanostructures}

There are nowadays a plethora of carbon nanostructures. Among them, one can mention:

- The Single walled carbon nanohorns (SWNHs): they are typically constituted by tubes of about 2 to $5 \mathrm{~nm}$ of diameter and 30 to $50 \mathrm{~nm}$ long, which associate with each others to give rise to round-shaped aggregates of $100 \mathrm{~nm}$ of diameter. Their large surface areas and inner nanospaces are of great importance since they ensure a great affinity, for example, with organic electron donors. They are especially good for hydrogen storage and electric field emission.

- The Carbon nanosheets (CNs), they are known as field emission source for high emission.

- The Carbon nanoporous are carbon "balls" often linked together as granular electuary waiver some holes between which shows an amorphous structure. Their images reveal nanopores distribution all around the carbon nanoporous balls.

- $\quad$ The Carbon nanoparticles (CNPs) which are characterized by an average diameter of 80 nanometers. They have in general catalyst particles encapsulated in.

- The Carbon nanobuds (CNBs): they are carbon nanostructures in which fullerenes are covalently attached to outer sidewall of CNTs. It is a hybrid material with useful properties, both of fullerenes' and CNTs'. CNBs have been found to be exceptionally good for field emitter.

\section{Synthesis of carbon nanotubes by chemical vapour deposition CVD}

Many applications as electronics' need CNTs with high density, high quality, and good alignment as prerequisites, because these qualities lead to stable parameters of the devices. And that is why special efforts are done in synthesized techniques since decades. Most of the existing models for the catalytic CVD growth of nanotubes are based on a mechanism formulated several years before the official CNTs discovery (1991), proposed by Baker (Baker et al.,1972), The CNSs growth method using a direct current plasma and hot 
filaments-enhanced catalytic chemical vapour deposition process has been fully described by Cojocaru (Cojocaru, 2003). Chemical vapour deposition can produce larger quantities of CNSs. CVD of hydrocarbons over metallic catalysts yields the carbon nanotubes and other carbon filaments with diameter changed from 1 to $100 \mathrm{~nm}$ depending on the synthesis operating parameters as temperature, kind and size of catalyst (Ivanov et al., 1994). A reactant gas containing carbon is inserted into a vacuum chamber at a given temperature between 600 and $1000 \mathrm{~K}$. In the presence of an appropriate transition metal (TM) catalyst, the gas is decomposed and, in the convenient conditions of temperature and pressure, the carbon can feed the growth of CNSs. Nanostructures grown by CVD are usually not straight and contain some defects.

There are many variants of CVD. We present the one known as Hot Filament Plasma Enhanced Catalytical Chemical Vapour Deposition (HF PE CCVD).

\subsection{Substrate preparation}

The substrate is prepared by the deposition of a $\mathrm{SiO}_{2}$ layer (thickness $8 \mathrm{~nm}$ ) by a Distributed Electron Cyclotron Resonance (DECR) plasma process on a $\mathrm{Si}(100)$ sample (Sb n-doped with $\rho=3 \mathrm{~m} \Omega . c \mathrm{~m}$; size $\left.8.5 \times 6 \times 0.245 \mathrm{~mm}^{3}\right)$. $\mathrm{SiO}_{2}$ is evaporated on $\mathrm{Si}(100)$ for two main reasons: it is a protective barrier layer that prevents the formation of transition metal silicide through direct interaction with silicon, and $\mathrm{SiO}_{2}$ is a non-wetting substrate that is convenient for transition metal (TM) diffusion and aggregation. However the thickness of the $\mathrm{SiO}_{2}$ layer must be thin enough to allow electron conduction through tunnelling for field emission measurements. This sample $\mathrm{SiO}_{2} / \mathrm{Si}(100)$ is then transferred into a stainless steel Ultra High Vacuum (UHV) preparation chamber (base vacuum 10-10 mbar) where TM consisting of Co or Fe or a mixture of them is evaporated without air removal. TM is evaporated with an OMICRON EFM3 effusive source at a pressure within 7-10.10-10 mbar on the sample heated at $925 \pm 20 \mathrm{~K}$ during $30 \mathrm{~min}$. The flux rate at $973 \mathrm{~K}$ is estimated to $0.025 \mathrm{~nm}$ of equivalent layer per minute from an in situ X-ray photoemission spectroscopy (XPS) analysis. In other cases the transition metal is deposited by sputtering (Cojocaru \& Le Normand, 2006).

\subsection{CNTs growth by the DC HF CCVD}

After the catalyst evaporation on the substrate, the whole is further transferred into an UHV CVD chamber for the growth of the carbon nanotubes and nanostructures in general at base pressure lower than 10-9 mbar (Fig. 12). The gas mixture $100 \mathrm{sccm}$ (Standard cubic centimetres per minute) $\mathrm{C}_{2} \mathrm{H}_{2}: \mathrm{H}_{2}: \mathrm{NH}_{3}$ is thermally activated by hot filaments up to a power $\mathrm{P}_{\mathrm{f}}$ around $150 \mathrm{~W}$ and kinetic energy-activated by polarisation between tantalum grid electrodes with the cathode grid in front of the sample at $V_{p}=-300 \pm 10 \mathrm{~V}$. The discharge is ignited and stabilized by the electron emission of the hot filaments. This ensured a high concentration of ionic species as well as activated radicals in front of the sample. A small additional negative extraction voltage of about $\mathrm{V}_{\mathrm{e}}=10 \mathrm{~V}$ is applied to the sample, which allowed withdrawing a controlled current of ionic species onto the sample $\left(\mathrm{I}_{\mathrm{e}}\right)$, with extraction power $\mathrm{P}_{\mathrm{e}}=\mathrm{I}_{\mathrm{e}}{ }^{*} \mathrm{~V}_{\mathrm{e}}$. The temperature which value is $973 \mathrm{~K}$ is controlled and regulated by an independent infrared heater set on the rear side of the sample. A Pt/PtRh thermocouple is contacted the rear side of the sample during the temperature rise. This thermocouple is switched off when the polarisation is started. The contact is then used to 
monitor the electric current onto the sample due to the discharge. The sequences of deposition are the following: the sample is first heated under vacuum $(10 \mathrm{~K} / \mathrm{min}, 573 \mathrm{~K}, 10$ $\mathrm{min})$. Then the temperature is risen to $973 \mathrm{~K}(10 \mathrm{~K} / \mathrm{min} ; 40 \mathrm{~min})$ in a $\mathrm{H}_{2}$ atmosphere at 15 mbar. The main growth parameters are shown on Table 1.

The Acetylene and ammonia were introduced. Subsequently the primary discharge and the extraction discharge onto the sample are adjusted to the desired values. The extraction current $\mathrm{I}_{\mathrm{e}}$ is set constant throughout the deposition process.

To stop the CNSs growth, the acetylene, the polarisation, the filaments and finally the hydrogen feed through are subsequently switched off. The growth mechanism of CNSs (CNTs, CNFs) occurs cleanly in a process constituting in three steps (Figs 13 \& 14):

a. the adsorption and the decomposition of hydrocarbon species such as $\mathrm{CH}_{4}, \mathrm{C}_{2} \mathrm{H}_{4}, \mathrm{C}_{2} \mathrm{H}_{2}$,

b. the diffusion of carbon through metallic particles and,

c. the extrusion for obtaining the graphitic walls.

\section{DC-HF-CCVD set up}

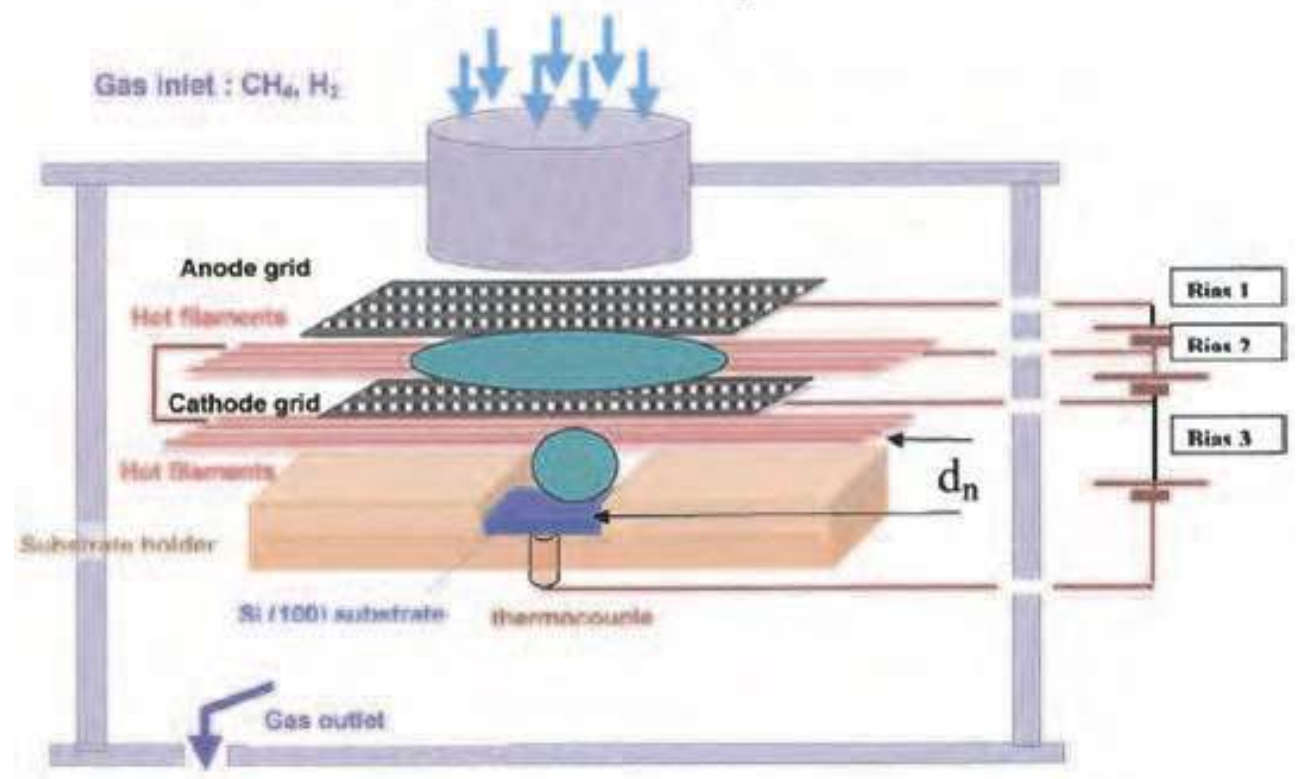

Fig. 12. Representation of the DC HF CCVD system, the distance $d_{n}$ between the substrate and the bottom of couple filaments is defined. The primary plasma between the cathode and the anode and the extraction plasma above the substrate are displayed in full. 
(a)
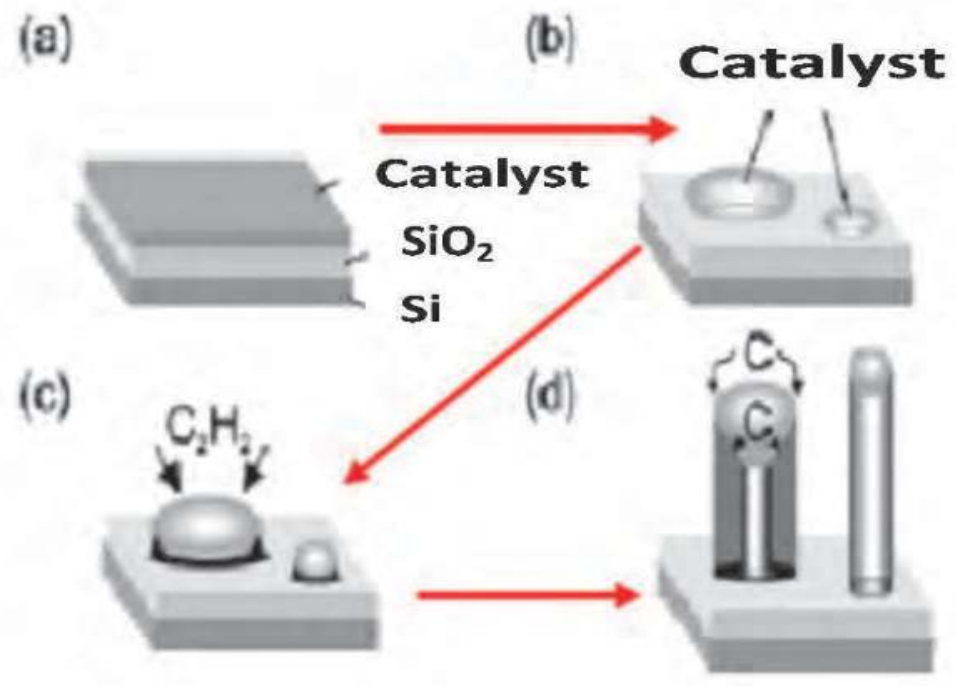

Fig. 13. Representation of the growth mechanism in DC HF CCVD process. (a) is the substrate consisting in silicon, silicon dioxide and catalyst (Co), (b) formation of calyst particles, (c) diffusion of carbon through catalyst particles (d) formation of graphitic walls.

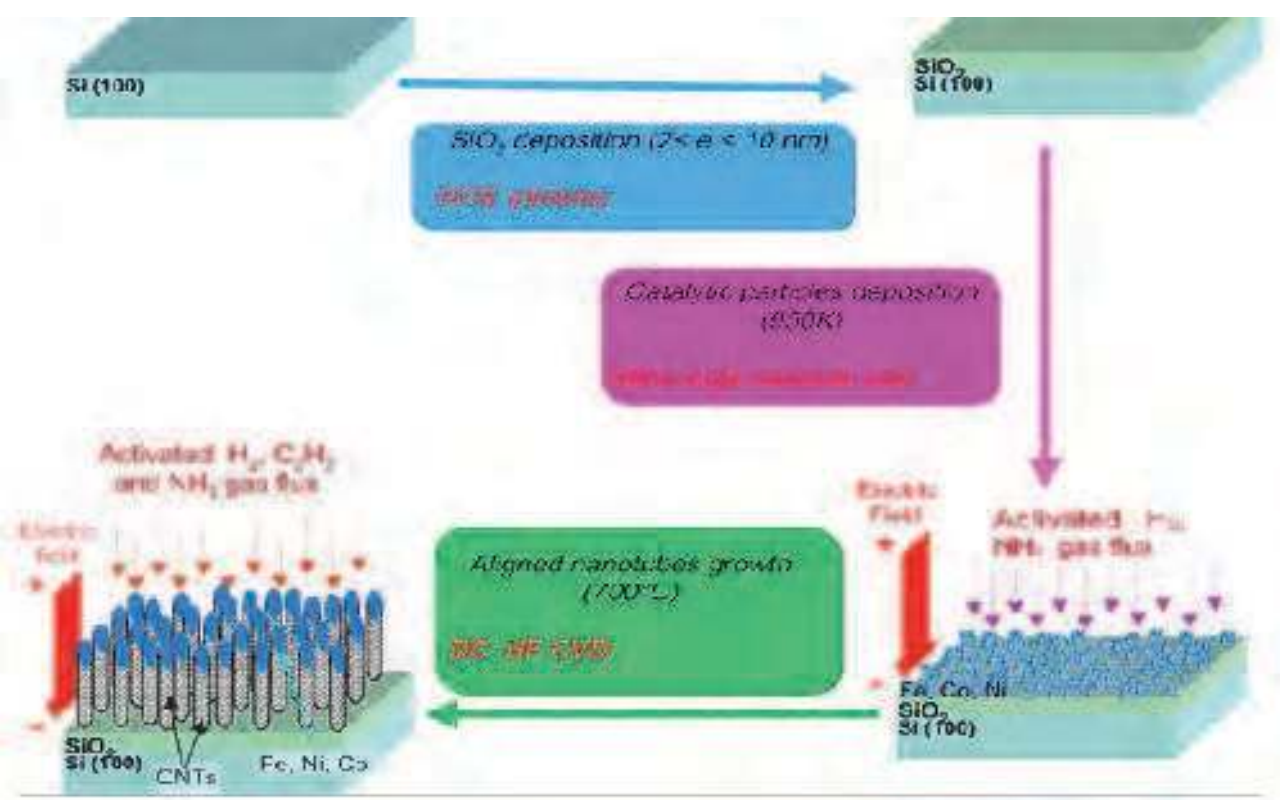

Fig. 14. Sketch of the steps of substrate preparation and CNTs growth in HF PE CCVD. 
According to the nature of the hydrocarbon, the mode of deposition of the catalyst as well as the pressure of the gas mixture, the temperature, the hot filaments power and the plasma power, different carbon nanostructures are allowed to grow as shown in Table 2 (Eba Medjo et al., 2009a). The growth process starts after the substrate elaboration and proceeds in the vacuum chamber: The first step is thermal reduction that occurs at temperature between 30 and $700^{\circ} \mathrm{C}$.

\begin{tabular}{|c|c|c|c|}
\hline $\mathrm{NH}_{3}(\%)$ & 0 & 1 & 3 \\
\hline Temperature substrate (K) & 973 & 973 & 973 \\
\hline $\mathrm{C}_{2} \mathrm{H}_{2}+\mathrm{NH}_{3}+\mathrm{H}_{2}(\mathrm{sccm})$ & 100 & 100 & 100 \\
\hline $\mathrm{C}_{2} \mathrm{H}_{2}(\%)$ & 20 & 20 & 20 \\
\hline $\mathrm{p}(\mathrm{mbar})$ & 15 & 15 & 15 \\
\hline $\mathrm{t}$ (minutes) & 15 & 15 & 15 \\
\hline $\begin{array}{c}\text { Activation power of the filaments (W) [Temperature of the } \\
\text { filaments (K)] }\end{array}$ & 150 & 150 & 150 \\
\hline$d_{n}(\mathrm{~mm})$ Substrate-Filaments distance & 5 & 5 & 5 \\
\hline $\mathrm{V}_{\mathrm{p}}^{*}(\mathrm{~V})$ DC activation & 310 & 300 & 295 \\
\hline $\mathrm{I}_{\mathrm{p}}{ }^{*}(\mathrm{~mA})$ DC activation & 3.75 & 11 & 16 \\
\hline $\mathrm{V}_{\mathrm{e}}^{* *}(\mathrm{~V})$ extraction & 10 & 10 & 10 \\
\hline $\mathrm{I}_{\mathrm{e}}^{* *}(\mathrm{~mA})$ extraction & 2 & 2 & 2 \\
\hline
\end{tabular}

Table 1. Main experimental growth parameters of PE HF CCVD

\begin{tabular}{|c|c|c|c|c|c|c|c|c|}
\hline Sample & Catalyst & $\begin{array}{l}\text { TM deposition } \\
\text { Process }\end{array}$ & $\begin{array}{c}\text { Carbon } \\
\text { Nanostructure }\end{array}$ & $\mathrm{TM} / \mathrm{Si}$ & $\begin{array}{l}P_{f} \\
(W)\end{array}$ & $\begin{array}{c}\mathrm{Pe}_{\mathrm{e}} \\
(\mathrm{mW})\end{array}$ & $\begin{array}{c}\text { Pressure } \\
\text { (mbars) }\end{array}$ & $\begin{array}{c}\mathrm{T} \\
(\mathrm{K})\end{array}$ \\
\hline I Nanot24 & Co & Sputtering & $\begin{array}{c}\text { CNFs (graphene // } \\
\text { substrate) }\end{array}$ & / & 150 & 10 & 15 & 973 \\
\hline $\begin{array}{c}\text { II } \\
\text { Nanot29 }\end{array}$ & Co & Sputtering & $\begin{array}{l}\text { CNTs (poorly } \\
\text { oriented) }\end{array}$ & / & 150 & 30 & 15 & 973 \\
\hline $\begin{array}{c}\text { III } \\
\text { Nanot30 }\end{array}$ & Co & Evaporation & $\begin{array}{c}\text { CNFs (graphene } \perp \\
\text { substrate) }\end{array}$ & 0.33 & 150 & 30 & 15 & 973 \\
\hline $\begin{array}{c}\text { IV } \\
\text { Nanot31 }\end{array}$ & $\mathrm{Co}$ & Evaporation & CNTs & 0.87 & 150 & 30 & 15 & 973 \\
\hline $\begin{array}{c}\mathrm{V} \\
\text { Nanot36 }\end{array}$ & Co & Evaporation & CNPs & / & 100 & 20 & 15 & 973 \\
\hline $\begin{array}{c}\text { VI } \\
\text { Nanot42 }\end{array}$ & Co & Evaporation & $\begin{array}{l}\text { CNTs (highly } \\
\text { oriented) }\end{array}$ & / & 145 & 20 & 15 & 1083 \\
\hline VII FLN1 & Co & Evaporation & $\begin{array}{l}\text { CNTs (medium } \\
\text { oriented) }\end{array}$ & / & 140 & 20 & 15 & 973 \\
\hline $\begin{array}{c}\text { VIII } \\
\text { FLN2 }\end{array}$ & $\mathrm{Co}-\mathrm{Fe}$ & Evaporation & $\begin{array}{l}\text { CNTs (highly } \\
\text { oriented) }\end{array}$ & / & 140 & 20 & 15 & 973 \\
\hline IX FLN4 & Co & Evaporation & CNWs & / & 140 & 20 & 5 & 973 \\
\hline
\end{tabular}

Table 2. Carbon nanostructures grown on $\mathrm{SiO}_{2}(5 \mathrm{~nm}) / \mathrm{Si}(100)$ substrate and main preparation characteristics. Other conditions are: $100 \operatorname{sccm~} \mathrm{C}_{2} \mathrm{H}_{2}: \mathrm{H}_{2}: \mathrm{NH}_{3}$ with relative content 20:79:1, filaments- substrate distance is 5mm; gas flow: $100 \mathrm{sccm}$. 
At this step, the plasma is not yet activated. The formation of TM particles begins. The second step is the catalyst reduction. It takes place at $700^{\circ} \mathrm{C}$ under the hot filaments thermal activation. With the activated gas flux of $\mathrm{H}_{2}$ and $\mathrm{NH}_{3}$, particles are formed. The HF PE CCVD system, the different steps of substrate preparation and CNSs growth are presented in figs 12, 13 and 14 (Cojocaru, 2003; Eba Medjo et al., 2009b). The last step is the growth of the CNSs after the addition of the acetylene and ammonia gas always. From the technologic point of view, CNTs synthesis aims to optimize field emission and implies the optimization of CNTs films growth in forms of bulk aligned and oriented perpendicularly on a plane substrate. To satisfy these requirements, methods of synthesis by chemical vapour deposition enhanced catalytically by using plasma and hot filaments are considered to be the most versatile. The presence of $\mathrm{TM}(\mathrm{Fe}, \mathrm{Co}, \mathrm{Ni})$ as catalysts spread on the substrate allows controlling local nucleation deeply for oriented CNTs.

The choice of transition metals is due to the metal-carbon diagram phase. In the range of temperatures used $\left(700-1200^{\circ} \mathrm{C}\right)$, carbon has a finite solubility in these metals, favour the formation of metal-carbon solid solution, segregation and further precipitation (Helveg et al., 2004). Preparation of metallic particles distribution (size, orientation, density...) is a crucial step of the overview process because the density and the size of TM particles control the size and the distribution of CNTs. To obtain a uniform spatial distribution of TM particles, a proceeding of metal evaporation in situ under ultra vacuum under and lower temperature on $\mathrm{SiO}_{2} / \mathrm{Si}(100)$ has been performed (Fig. 14).

\section{Other carbon nanotubes synthesis methods}

\subsection{Arc discharge}

The arc discharge method, initially used for producing fullerenes, is the most common and perhaps easiest way to produce CNTs as it is rather simple (Iijima, 1991). The most perfect multiwall carbon nanotubes are produced using the arc-discharge evaporation of graphite in an inert atmosphere (Ebbesen \& Ajayan, 1992). Arc discharge apparatus is presented in the following figure (Fig. 15).

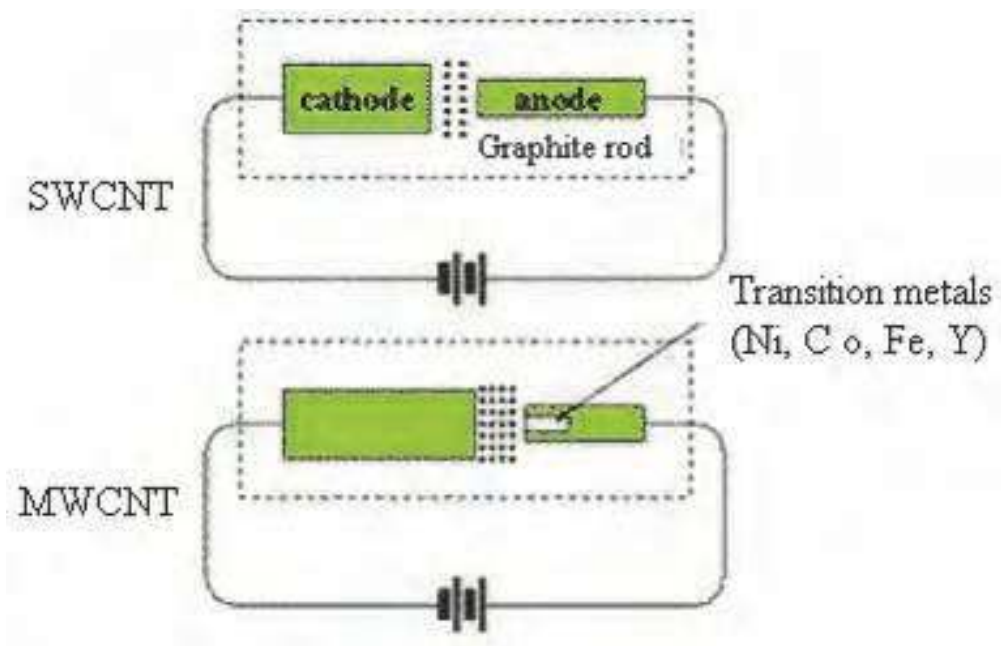

Fig. 15. Schematic diagram of Arc discharge apparatus. 
However, it is a technique that produces a complex mixture of components and requires further purification to separate the CNSs from the soot and the residual catalytic metals present in the crude product. This method creates CNSs through arc-vaporization of two carbon rods placed end to end, separated by approximately $1 \mathrm{~mm}$, in an enclosure that is usually filled with inert gas at low pressure. According to recent investigations, it is also possible to create CNSs with the arc method in liquid nitrogen. A direct current of 50 to 100 A, driven by a potential difference of approximately $20 \mathrm{~V}$, creates a high temperature discharge between the two electrodes. The discharge vaporizes the surface of one of the carbon electrodes, and forms a small rod-shaped deposit on the other electrode.

\subsection{Plasma arcing}

This method was the first for producing CNTs in reasonable quantities. It consists of applying an electric current across two carbonaceous electrodes in an inert gas atmosphere. It involves the evaporation of one electrode as cations followed by deposition at the other electrode. This plasma-based process is analogous to the more familiar electroplating process in a liquid medium. CNTs are formed by plasma arcing of carbonaceous materials, particularly graphite. The fullerenes appear in the soot that is formed, while the CNTs are deposited on the arcing in the presence of cobalt with a $3 \%$ or greater concentration. However, when cobalt is added as a catalyst, the nature of the product changes. The mechanism by which cobalt changes this process is still unclear.

\subsection{Ball milling}

Essentially, this method consists of placing graphite powder into a stainless steel container along with four hardened steel balls. The container is purged, and argon is introduced. The milling is carried out at room temperature for up to 150 hours. Following milling, the powder is annealed under an inert gas flow at temperatures of $1400{ }^{\circ} \mathrm{C}$ for six hours. The mechanism of this process is not well known, but it is thought that the ball milling process forms nanostructures nuclei, and the annealing process activates carbon nanotubes growth.

\subsection{Laser ablation or laser vaporization}

Laser ablation near the surface of the target creates an almost continuous inflow of hot carbon atoms and ions, into the experimental chamber. The shock wave generated by each pulse rapidly decelerates in the ambient gas atmosphere and further propagation of hot atoms proceeds by diffusion, finally forming a mixture of carbon and filling gas with some average density and temperature. The density and temperature of this mixture change with the distance from the target. Depending on the masses of ablated atoms and the atoms of the filling gas, the processes of energy exchange will occur at different rates. If the masses of colliding atoms are comparable, the carbon can lose a significant part of its energy in a single collision. Hence, efficient energy equilibration occurs after several collisions: the carbon vapour is cooling down and the argon gas is heating up.

On the basis of kinetic considerations, the following scenario for carbon nanotubes formation in a carbon-argon mixture created by high repetition rate laser ablation has been suggested. Initially, the chamber gas is at ambient temperature. The continuous inflow of hot carbon increases the temperature in the mixture. When the carbon vapour temperature and the number density reach the level where the probability of carbon-carbon attachment becomes significant, the formation of carbon nanotubes begins. The carbon consumption rate during 
this formation process significantly exceeds the evaporation rate due to laser ablation. Therefore, the carbon density rapidly decreases to the value where the formation process terminates. Thus, the ablation rate, target parameters, pressure and the ambient gas determine the formation time, and accordingly, the size of the nanotubes. This technique produces CNTs of almost as high quality as arc discharge. The temperatures reached are included in the range of 1200 to $2000 \mathrm{~K}$, which explains the residual difference in graphitization.

\section{Carbon nanotubes contamination}

Fig. 16 shows two CNTs XANES spectra with drastic differences. If the defects like: topological defects in the grapgene sheets (the occurrence of pentagons and heptagons), the $s p^{3}$ hybridized carbons atoms and incomplete bonding that have slight changes that can be neglected, are not taken into account, it is observed that some features present in Fig. 16 (a) are not found in Fig. 16 (b), especially peaks K and L, and are replaced by $\mathrm{P}, \mathrm{P}^{\prime}$ and $\mathrm{P}^{\prime \prime}$ (Eba Medjo et al, 2009a).

These new peaks are not intrinsic to CNTs. They are the result of contamination which can be considered as accidental adsorption of atoms, molecules or radical compounds, in agreement with SEM and TEM analysis, where it is not found bundles of CNTs as it may be, according to literature. Among the reasons of the presence of non-intrinsic features in XANES spectra is the presence of TM particles as proved by SEM and TEM. Actually, it is known that the features attributed to the so-called free-electron-like interlayer (FELI) states in the graphite and other carbon nanostructures are also due to contamination (Kuznetsova et al., 2001). The peaks P, $\mathrm{P}^{\prime}$ and $\mathrm{P}^{\prime \prime}$ are assigned to adsorbed potassium atoms according to features parameters (Sasaki, 1984; Eba Medjo et al., 2009a). The samples are constituted of different kinds of CNSs. Their behaviour with those contaminants is of great importance and helps to distinguish them. XANES spectra of those samples present many differences. When analyzing unannealed spectra, some of them do not display $\mathrm{P}, \mathrm{P}^{\prime}$ and $\mathrm{P}^{\prime \prime}$ features. In Fig. 16, the comparison of chemical inertness of unannealed samples shows an important difference.

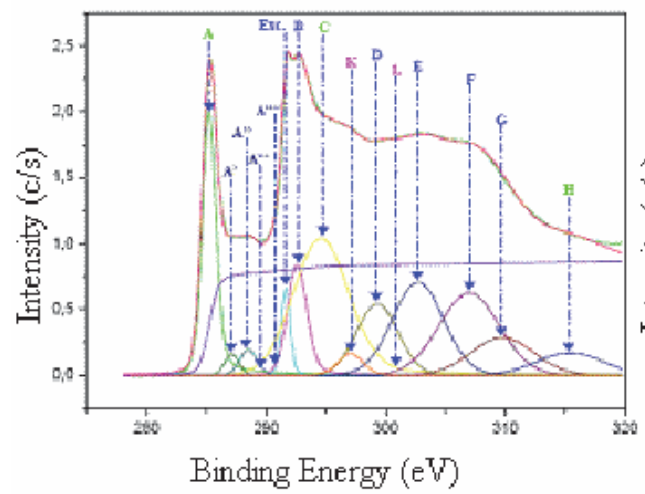

(a) MWCNT

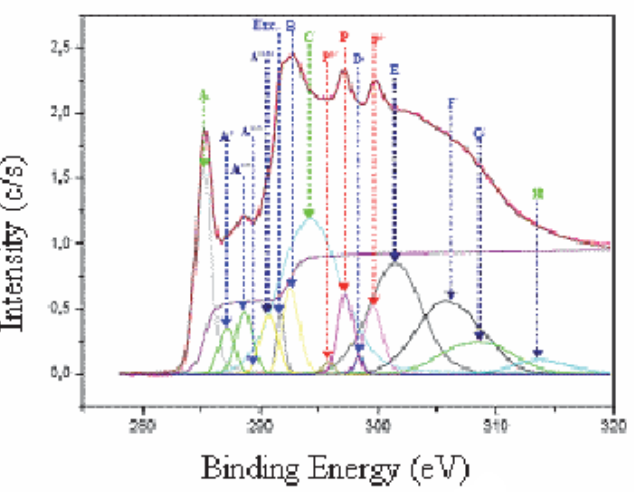

(b) SWCNT

Fig. 16. CNTs experimental and calculated superimposed XANES spectra at grazing incidence from unannealed samples: (a) without potassium contamination, (b) with potassium contamination. 
In (a) there is no potassium contamination, while in (b), the intensity of potassium peaks is considerable. The spectrum (a) belongs to MWCNT which is not chemically as reactive as SWCNT (b) with a great reactivity and can easily establish chemical bond. The chemical inertness is important for CNSs that gain less $s p^{3}$ character during their growth or the curvature, in other words, those with a great diameter or small curvature. The comparison of XANES spectra of unannealed samples shows another important difference. This difference is due once more to the varying behaviour of carbon nanostructures samples in presence of contaminants. Fig. 16 (a) presents less contamination of potassium than Fig. 16 (b). In the typical case of CNTs, when the outer diameter is important, the CNTs are less chemically active and therefore are not favoured to establish chemical bonds. The adsorption contribution to the signal is observed at the contaminants adsorbed region in the free-electron-like interlayer states band, when no previous annealing of the sample is realized before recording the spectra (Kuznetsova et al., 2001).

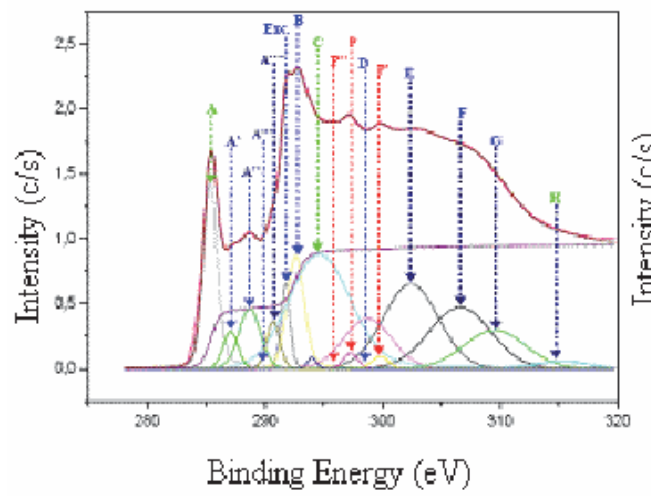

(a) MWCNT

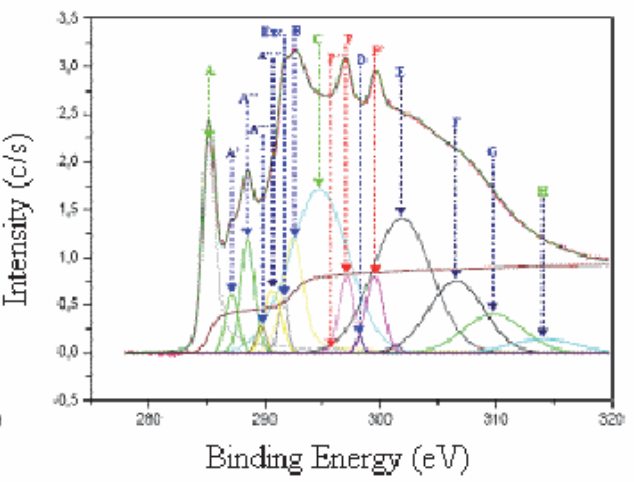

(b) SWCNT

Fig. 17. Contaminated CNTs experimental and calculated superimposed XANES spectra at normal incidence from annealed $\left(550^{\circ} \mathrm{C}\right)$ samples: (a) sample is sensitive to thermal annealing; (b) sample is less sensitive to thermal treatment.

But the sensitivity to thermal treatment is not uniformed for each sample, as shown in Fig. 17. The sample in Fig. 17 (a) is more sensitive to thermal annealing than the one whose XANES spectrum is presented in Fig. 17 (b). This difference is assigned to the curvature effect, which is relative to the outer diameter and the $s p^{2} / s p^{3}$ ratio of the CNT. The contamination is due to atomic, radical or molecular adsorption of many species present or formed during synthesis phase, such as oxygen $(\mathrm{O})$; water $\left(\mathrm{H}_{2} \mathrm{O}\right)$ and TM. The spectral features $\mathrm{P}, \mathrm{P}^{\prime}$ and $\mathrm{P}^{\prime \prime}$ are assigned to potassium $(\mathrm{K})$ along with the IP of $\mathrm{K}, \mathrm{L}$...levels of the element. This potassium present in samples might come from the beam line on the synchrotron station.

\section{Carbon nanotubes characterization}

Because of contamination and defects occurring during synthesis phases and the possibility to obtain many kinds of CNSs, it is basic to characterize each nanostructure synthesized. 
Characterization helps to avoid false results, confusions or wrong conclusions concerning nanostructures and their properties, and of course their applications. There are many techniques of CNSs characterization. The most utilize are electron microscopy which is a powerful technique in the sense that, it can be directly correlated to the atomic-scale microstructure of the carbon nanostructure with its physical properties, thus providing a characterization of the morphology and the nanostructure (Somani \& Umeno, 2007).

Raman spectroscopy is also used to identify the hybridization of carbon atoms in each of the carbon nanostructures obtained. So this spectroscopy is very important in the characterization of CNSs. XAS recorded on the carbon K-edge is a powerful tool to provide chemicallyselective information on the local environment around carbon in solid materials, like diamond, amorphous carbons nitride, amorphous graphitic carbon and CNSs (Eba Medjo et al., 2009a). The technique is also suitable to probe the adsorption of functional organic molecules with information both on the distance and the orientation of bonds (Kuznetsova et al., 2001; Eba Medjo et al., 2009b). This property is due to the angular dependence of the absorption transitions. The angular dependence has been reported on graphite since a long time (Rosenberg et al., 1986) and the analysis has been further refined both on an experimental and a theoretical points of view. The $\pi^{*}$ antibonding state corresponds to the out-of-plane bonds in the $\mathrm{sp}^{2}$ bonding configuration and exhibits strong polarization dependence. According to the absorption process, the signal is maximum when the direction of the electric field of the incident light matches the direction of unfilled orbitals.

The CNSs obtained are of four kinds. When the catalyst is prepared by sputtering and the plasma power rather low, the CNFs can grow with graphene sheets axis parallel to the surface. Strong adhesion of the catalyst to the substrate and low energy ions can explain this mode of growth. Carbon nanoparticules (CNPs) are prepared when the power of the hot filaments is low. The growth of graphene layer seems impossible. The ammonia introduced in gas mixture during the synthesis has three concentrations, which are 0,1 and $3 \%$ respectively. The acetylene concentration remained at $20 \%$. Hydrogen is the third and last gas in the mixture. The activation power of the filaments is $150 \mathrm{~W}$ and yields the filaments temperature of $2100 \mathrm{~K}$. The distance $d_{n}$ between the substrate and the bottom couple of filaments is $5 \mathrm{~mm}$. Under these synthesis conditions it is possible to control the nature of the carbon nanostructures synthesized in agreement with literature (Cojocaru \& Le Normand, 2006). The four types of CNSs obtained are, according to SEM images (Table 1):

- Carbon nanowalls (CNWs),

- Carbon nanoparticules,

- Carbon nanofibers, with graphene sheet parallel and perpendicular to the substrate respectively and,

- Carbon nanotubes, with poor, medium and high orientation, respectively (Eba Medjo, 2011).

Within the medium value of the plasma power (Table 2), as shown with SEM study, carbon nanotubes are yet grown. Transmission electron microscope is employed to improve the characterization of the nanostructures by SEM. These samples however display different mutual orientations. The highly oriented films are obtained under optimized conditions and poorly and medium oriented films are also obtained, showing more defects. One of the very important observations is that each carbon nanostructure grown has TM nanoparticles at its top. One observes, TM particles inside tubules, on tubes walls coming from growth process by VLS (Vapour, Liquid and Solid) mode (Helveg et al., 2004). TEM observations show at the same time that the surface of the nanotubes exhibits an amorphous structure due to wall surface defects. 
The outer diameters of the CNTs are directly determined from TEM images with high accuracy. Thus the mean outer diameter is 15 nanometers the smallest being $6 \mathrm{~nm}$ corresponding surely to SWCNTs or to a few number of walls. The upper values, may account for outer diameters of MWCNTs. The analysis of TEM images leads to extensive values of length, varying from 100 to 400 nanometers. The density of nanotubes spreads also in a very large range. It ranges between 350 to $1000 \mu \mathrm{m}$. Fig. 18 (c) shows CNTs completely detached from the substrate with catalyst particles on one end, and the graphitic end of attachment to the substrate. Unfortunately, TEM images of CNTs do not give the opportunity to determine the exact number of walls of each sample of CNTs.

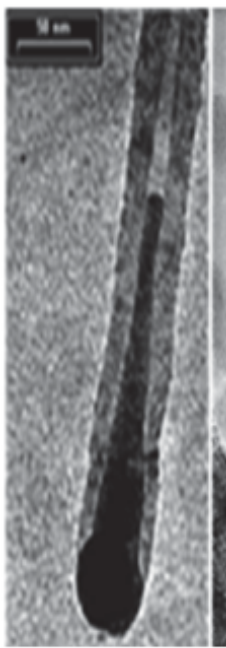

(a)

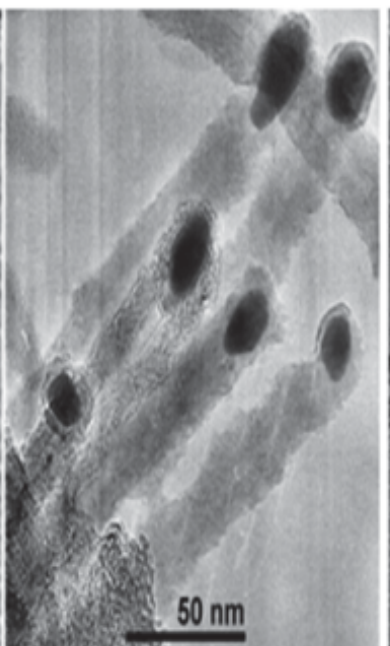

(b)

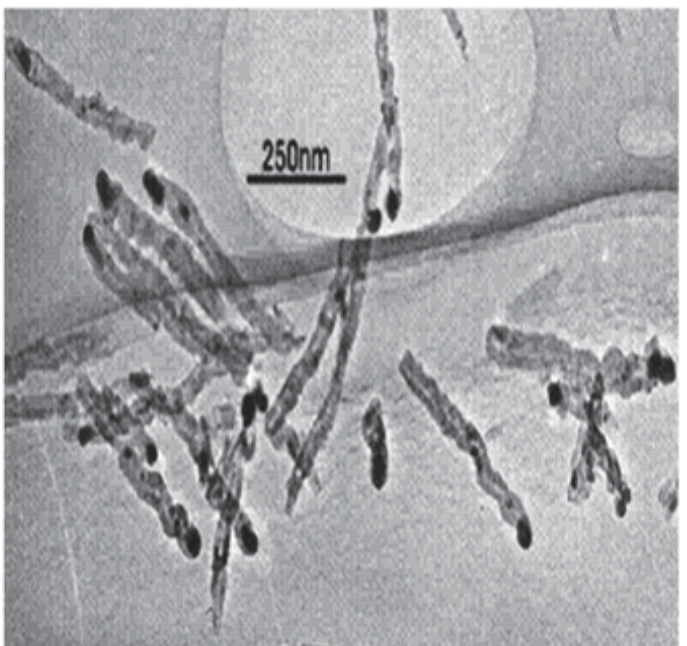

(c)

Fig. 18. CNTs TEM images with TM particles inserted at one end: (a) CNT highly oriented, (b) CNTs medium oriented and (c) CNTs sample synthesized with $1 \%$ of $\mathrm{NH}_{3}$. The two ends of each CNT are well observed detached from the substrate. One end has graphitic structure (half fullerene) and the other has a TM particle inserted in the tube.

This deficiency is the result maybe of defects mutually aligned CNTs of different densities are obtained, depending on the ammonia concentration in the reactive gas mixture. A perfect $\mathrm{CNT}$ is an abstraction, because the hexagonal structure $\mathrm{sp}^{2}$ forms layers with different types of alterations as shown in literature. These alterations can come from the growth mode, from the deposit on a substrate, or they can be the result of a heat or chemical treatment (Ebbesen \& Takada, 1995). An important consequence of these defects on surface morphology that needs to be pointed out is the roughness of CNTs surface.

The defects are in general very important because they can modify the electronic properties of the nanostructure, and so, can be seen as a feature that can influence nanostructures applications. Generally, a defective site has a high chemical reactivity, in other words, this site is chemical reactions favoured (Eba Medjo et al., 2009a). Thus CNTs with poor orientation and those many defects are not appropriate for applications in field emission devices, but can have other important applications like chemical functionalization. In most cases, TEM investigations and seldom in SEM's, it is shown that the catalyst metal particles 
are attached to the nanostructures top end Fig. 18, or found inside or at last in the sidewalls. According to the aimed applications, the presence of ultrafine cobalt or cobalt/iron nanoparticles of various diameters at the top of CNTs can have negative effect. In particular in the case of emission of electrons or electric field, the particles need to be carried off. Thus, the purification of nanostructures needs to be one of crucial parts of synthesis process. TEM gives direct insight into the structure of carbon nanomaterials and can help to identify the material or the phase correctly. Without observations by TEM, one may lead to wrong or incorrect conclusions.

It is the most important and most reliable technique for correctly identifying the nature and the form of carbon nanomaterials, in spite of the fact that the information that can be extracted is not straight-forward, since the preparation of TEM samples may mask observation of the nanostructures of lower size, or the possibility of the projection of artefacts. TEM has become useful for in situ microscopy, for observing dynamic processes at the nanoscale measurements which directly correlate physical properties with structures, holographic imaging of electric and magnetic fields, quantitative chemical mapping at subnanometer resolution and for ultra-high resolution imaging.

\begin{tabular}{|c|c|c|c|c|c|}
\hline \multicolumn{6}{|c|}{ CNTs main features at normal incidence for FLN1_OE56 } \\
\hline $\begin{array}{c}\text { Peak } \\
\text { Names }\end{array}$ & Peaks & \begin{tabular}{|c|} 
Binding \\
energy $(\mathrm{eV})$
\end{tabular} & $\begin{array}{c}\text { Kinetic } \\
\text { energy }(\mathrm{eV})\end{array}$ & $\begin{array}{l}\text { Intensity } \\
(\mathrm{c} / \mathrm{s})\end{array}$ & $\begin{array}{c}\text { Final-state band and Brillouin- } \\
\text { zone }\end{array}$ \\
\hline $\mathrm{A}$ & 285.50 & \begin{tabular}{|l|}
285.20 \\
\end{tabular} & 1199.20 & 3.55 & $\pi_{0}$ near $\mathrm{Q}$ \\
\hline $\mathrm{A}^{\prime}$ & 286.53 & 287.14 & 1197.26 & 0.24 & $\begin{array}{l}\text { free-electron-like interlayer } \\
\text { states + adsorption }\end{array}$ \\
\hline $\mathrm{A}^{\prime \prime}$ & 288.40 & 288.50 & 1195.90 & 0.40 & $\begin{array}{l}\text { free-electron-like interlayer } \\
\text { states }+ \text { adsorption }\end{array}$ \\
\hline $\mathrm{A}^{\prime \prime \prime}$ & 288.70 & / & / & / & $\begin{array}{l}\text { free-electron-like interlayer } \\
\text { states }+ \text { adsorption }\end{array}$ \\
\hline $\mathrm{A}^{\prime \prime \prime \prime}$ & 290.70 & / & / & / & $\begin{array}{l}\text { free-electron-like interlayer } \\
\text { states }+ \text { adsorption }\end{array}$ \\
\hline C-H Exc. & 291.76 & 291.65 & 1192.75 & 0.68 & Exciton \\
\hline $\mathrm{B}$ & 292.65 & 292.57 & 1191.83 & 1.93 & $\sigma_{1}, \sigma_{2}: \Gamma \rightarrow Q$ \\
\hline $\mathrm{C}$ & 295.50 & 294.50 & 1189.90 & 6.54 & $\pi_{0}$ or $\pi_{1}$ near $\Gamma$ \\
\hline $\mathrm{D}$ & 297.80 & 297.06 & 1187.34 & 0.45 & $\sigma_{3}-\sigma_{6}: \mathrm{Q} \rightarrow \mathrm{P}$ \\
\hline $\mathrm{E}$ & 303.50 & 302.70 & 1181.70 & 3.83 & $\sigma_{7}$ near $\mathrm{Q}$ \\
\hline $\mathrm{F}$ & 307.50 & 306.97 & 1177.42 & 4.00 & $\sigma_{9}$ near $Q$ \\
\hline$G$ & 308.50 & 309.75 & 1174.65 & 1.90 & l \\
\hline $\mathrm{H}$ & 316.50 & 315.40 & 1169.00 & 1.20 & $\pi_{4}$ near $Q$ \\
\hline $\mathrm{I}$ & 329.00 & 329.00 & / & / & / \\
\hline $\mathrm{J}$ & 333.00 & 333.00 & / & I & 1 \\
\hline $\mathrm{K}$ & 295.40 & 297.05 & 1187.35 & 0.00 & $\pi_{0}$ or $\pi_{1}$ near $\Gamma$ \\
\hline $\mathrm{L}$ & 301.85 & 299.30 & 1185.10 & 2.40 & $\sigma_{6}-\sigma_{7}:$ near $Q$ \\
\hline & $(A+C+1$ & $+\mathrm{K}) /(\mathrm{B}+\mathrm{D}+\mathrm{H}$ & $\mathrm{E}+\mathrm{F}+\mathrm{G}+\mathrm{L})=($ & 778 & $\mathrm{R}=(\pi / \sigma)_{\text {tot }}$ \\
\hline
\end{tabular}

Table 3. Main features' parameters for CNTs XANES spectrum none contaminated by potassium at normal incidence from FLN1_OE56. 
The assignment of main features of the XANES spectra of CNTs at normal incidence is given by Table 3 and Table 4, with CNTs samples none potassium-contaminated and potassiumcontaminated respectively.

\begin{tabular}{|c|c|c|c|c|c|}
\hline \multicolumn{6}{|c|}{ Annealed and potassium-contaminated CNTs at normal incidence Nanot29_OE36 } \\
\hline $\begin{array}{l}\text { Peaks } \\
\text { Names }\end{array}$ & Peaks & $\begin{array}{c}\text { Binding } \\
\text { energy } \\
(\mathrm{eV})\end{array}$ & $\begin{array}{c}\text { Kinetic } \\
\text { energy }(e V)\end{array}$ & $\begin{array}{l}\text { Intensity } \\
\quad(\mathrm{c} / \mathrm{s})\end{array}$ & $\begin{array}{l}\text { Final-state band and } \\
\text { Brillouin-zone }\end{array}$ \\
\hline A & 285.50 & 285.20 & 1199.20 & 4.75 & $\pi_{0}$ near Q \\
\hline $\mathbf{A}^{\prime}$ & 286.53 & 287.31 & 1197.09 & 1.66 & $\begin{array}{c}\text { free-electron-like } \\
\text { interlayer states + } \\
\text { adsorption }\end{array}$ \\
\hline $\mathrm{A}^{\prime \prime}$ & 288.40 & 288.67 & 1195.73 & 2.12 & $\begin{array}{c}\text { free-electron-like } \\
\text { interlayer states + } \\
\text { adsorption }\end{array}$ \\
\hline $\mathrm{A}^{\prime \prime \prime}$ & 288.70 & 289.68 & 1194.72 & 0.03 & $\begin{array}{c}\text { free-electron-like } \\
\text { interlayer states + } \\
\text { adsorption }\end{array}$ \\
\hline$A^{\prime \prime \prime \prime}$ & 290.70 & 290.35 & 1194.05 & 2.10 & $\begin{array}{c}\text { free-electron-like } \\
\text { interlayer states + } \\
\text { adsorption }\end{array}$ \\
\hline C-H Exc. & 291.76 & 291.64 & 1192.76 & 0.73 & Exciton \\
\hline B & 292.65 & 292.40 & 1192.00 & 3.03 & $\sigma_{1}, \sigma_{2}: \Gamma \rightarrow Q$ \\
\hline $\mathrm{C}$ & 295.50 & 294.67 & 1189.73 & 14.40 & $\pi_{0}$ or $\pi_{1}$ near $\Gamma$ \\
\hline $\mathrm{D}$ & 297.80 & 298.30 & 1186.10 & 0.35 & 1 \\
\hline $\mathrm{P}^{\prime \prime}$ & 297.51 & 296.20 & 1188.20 & 0.17 & Potassium $\mathrm{L}_{2}$ level \\
\hline $\mathrm{E}$ & 303.50 & 301.85 & 1182.55 & 9.45 & $\sigma_{7}$ near Q \\
\hline $\mathrm{F}$ & 307.50 & 306.54 & 1177.86 & 5.78 & $\sigma_{9}$ near $Q$ \\
\hline$G$ & 308.50 & 310.00 & 1174.40 & 2.60 & / \\
\hline $\mathrm{H}$ & 316.50 & 314.30 & 1170.10 & 1.08 & $\pi_{4}$ near $Q$ \\
\hline I & 329.00 & 329.00 & / & / & / \\
\hline $\mathrm{J}$ & 333.00 & 333.00 & / & / & / \\
\hline $\mathrm{P}$ & 296.60 & 297.20 & 1187.20 & 1.16 & Potassium $\mathrm{L}_{2}, \mathrm{~L}_{3}$ level \\
\hline $\mathrm{P}^{\prime}$ & 299.62 & 299.37 & 1184.78 & 1.83 & Potassium $\mathrm{L}_{2}$ - $\mathrm{L}_{1}$ levels \\
\hline \multicolumn{5}{|c|}{$\mathrm{R}=\left(\mathrm{A}+\mathrm{C}+\mathrm{H}+\mathrm{P}^{\prime \prime}\right) /(\mathrm{B}+\mathrm{D}+\mathrm{E}+\mathrm{F}+\mathrm{G})=0.962$} & $\mathrm{R}=(\pi / \sigma)_{\text {tot }}$ \\
\hline
\end{tabular}

Table 4. Main features' parameters for annealed and potassium-contaminated CNTs samples XANES spectrum at normal incidence.

Understanding the growth mode of CNTs is among the imperatives in their characterization and can lead to the growth modelling. Two dominant growth modes are observed in SEM and TEM images, the tip growth mode and the base growth mode. In tip growth mode, the transition metal nanoparticle catalyzing the growth remains at the top. The adhesive forces between the substrate and the catalyst nanoparticles seem typically too small and the 
particles are lifted up as the CNTs grow. The process takes place till the temperature is upper, before the cooling.

When the adhesive forces between the catalyst TM particles and the substrate are very important, the catalyst nanoparticles are not lifted up by the growing CNT during the growth process. The TM particles remain at the bottom of the CNT. The parameters of each kind of growth mode have yet to be clarified, because the nature of the growth mode, whether tip or base, is basic for the knowledge and the choice of the growth mechanism aiming the way of various applications.

In the light of the XANES and HOPG spectra used as a starting point model, a prior annealing of the samples prevents the increase of intensity in the free-electron-like interlayer states region of the spectra, clarifying that these features are not intrinsic. The use of the OTP for CNTs, the parameter $R(\alpha)$, deduced from the fitting of the carbon K-edge X-ray absorption spectra by the ratio of the intensity of $\pi$-type features over $\sigma$-type features' one, is defined to determine more quantitatively the respective contributions of the $\sigma^{*}$ and $\pi^{*}$ transitions at incidence angle $\alpha$, is dual and completes electron microscopy characterization.

The parameter $R(\alpha)$ is expressed by the relation below:

$$
R(a)=\frac{A+C+H+K}{B+D+E+F+G+L}
$$

where A, B, C... are the main peaks assigned in the XANES spectrum of CNTs, or

$$
R(a)=\frac{\sum \pi}{\sum \sigma}
$$

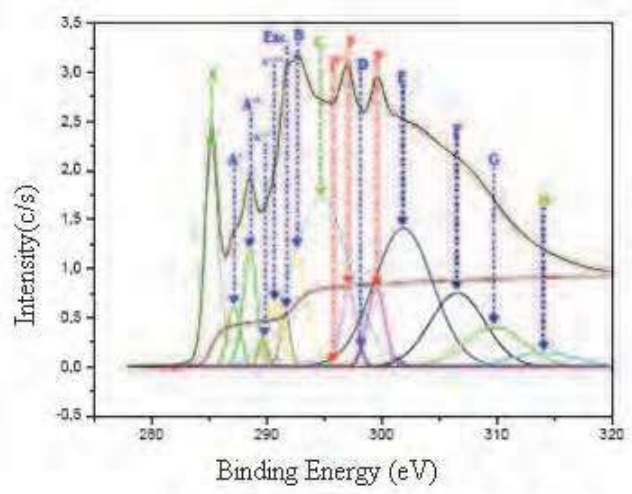

(a) XANES spectrum at NI

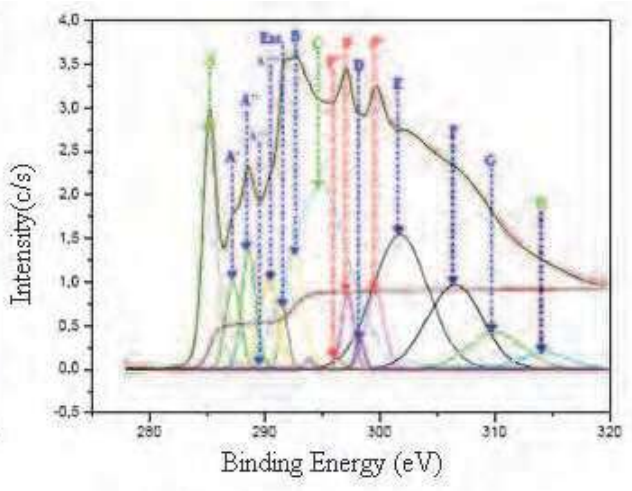

(b) XANES spectrum at GI

Fig. 19. Potassium contaminated CNTs experimental and calculated superimposed XANES spectra at normal incidence (NI) and at grazing incidence (GI).

The OTP can first of all give information about the orientation of the CNTs relatively to the substrate plan (Eba Medjo et al., 2009b). It can moreover inform about the contamination and by the way lead to quantitative properties of CNTs, as the ability to functionalization, 
the curvature and of course the number of walls (SWCNTs or MWCNTs) and chemical inertness (Eba Medjo et al., 2009a). As a general trend, the $R(\alpha)$ parameter values at normal incidence are greater than those at grazing incidence for CNTs contrary to those of the HOPG. The obtained CNTs are classified in three groups according to its values according to Table 5:

- Those with poor orientation,

- The other with medium range orientation,

- The last group which is formed of high oriented nanostructures.

For poorly oriented CNTs, the discrepancy between the two values (NI and GI) is too small. It increases towards high oriented CNTs according to Table 5 bellow.

\begin{tabular}{|c|c|c|c|}
\hline CNTs & CNTs1 & CNTs2 & CNTs3 \\
\hline NI & 0.778 & 0.962 & 0.975 \\
\hline GI & 0.732 & 0.883 & 0.665 \\
\hline$\Delta \mathrm{R}$ & 0.046 & 0.079 & 0.310 \\
\hline Observation & Poor Oriented & Medium Oriented & High Oriented \\
\hline
\end{tabular}

Table 5. The values of $R(\alpha)$ of three samples of CNTs. The discrepancy of these values at normal incidence (NI) and grazing incidence (GI) allows the classification of CNTs in: poor, medium and high oriented.

The comparison of XANES spectra of unannealed samples and annealed ones shows an important difference due to the variation of the behaviour of carbon nanostructures samples in presence of contaminants.

When the outer diameter is important (MWCNTs), the CNTs are less chemically reactive and therefore, are not favoured to establish chemical bonds. But when the outer diameter is too small (SWCNTs), the chemical reactivity is very important, and such CNST are appropriate for functionalization.

The adsorption contribution to the signal of the contaminants in the free-electron-like interlayer states region is observed when no previous annealing of the sample is realized. This absorption contribution is important at normal incident than at grazing incidence. But the sensitivity to thermal treatment is not uniform for each sample. The difference is assigned to the curvature effect which is relative to the outer diameter and the $s p^{2} / s p^{3}$ ratio of each CNT type. These results elucidate that the CVD is one of the best techniques for synthesis of the CNSs for multiple purposes till date.

\section{Single walled carbon nanotubes functionalization}

The functionalization of carbon nanotubes in general and single wall carbon nanotubes in particular is among the very actively discussed topics in nanoscience, because of the planned modification of properties is believed to open towards nanotechnology applications. Among the proposed applications closed to technical realization, recent developments of reliable methods for the functionalization of the SWCNTs provide an additional impetus to extend the scope of their application spectrum (Kuzmany et al, 2004). On top of their properties, SWCNTs possess high mechanical and chemical stability. This latter property is certainly advantageous from an application point of view. This attribute imposes chemical modification schemes, whereby new functions can be implemented that 
cannot otherwise be acquired by pristine nanotubes, and lead to the development of methods allowing selective and controlled functionalization. For most applications SWCNTs require functionalization, such as changing some of the graphite properties to make nanotubes soluble in different media, or attaching different radicals or even inorganic particles (Hirsch, 2002) for future utilization.

Functionalization methods of carbon nanotubes can be divided into two major groups:

- Endohedral functionalization which consist to fill SWCNTs with some colloidal suspensions. It can be done by exploiting the phenomenon of spontaneous penetration or by wet chemistry method of filling nanotubes (Pederson \& Broughton, 1992). SWCNTs are also filled by fullerenes. Sometimes, polymerization of fullerenes is done inside the SWCNTs.

- Exohedral chemical functionalization: its purpose is to attach covalently functional groups (-COOH for example) or metals in the outer walls of SWCNTs to make it soluble in different media. This kind of functionalization can be done via the main source of reactivity of the nanotubes which are: the curvature induce effect, the defects (misalignment) and the ends or the capping fullerene hemispheres. Among the most popular class of functionalization of nanotubes is the noncovalent exohedral functionalization, by wrapping SWCNTs in peptides or polymers for example.

There are various ways to functionalize SWCNT. Any functionalization of pristine CNTs is made to change properties such as inertness, solubility, conductivity, magnetism, etc., thus making nanotubes applicable for different purposes. So functionalization of functional groups creates new class of carbon nanomaterials with new properties. In principle functionalization is possible from the inside of the nanotubes and on the side-walls. It will help to separate metallic SWCNTs to semiconducting ones. This separation is of basic important in the fabrication of Carbon nanotube field effect transistor (CNTFET). Fig. 20 shows functionalization of SWCNT trough oxidation.

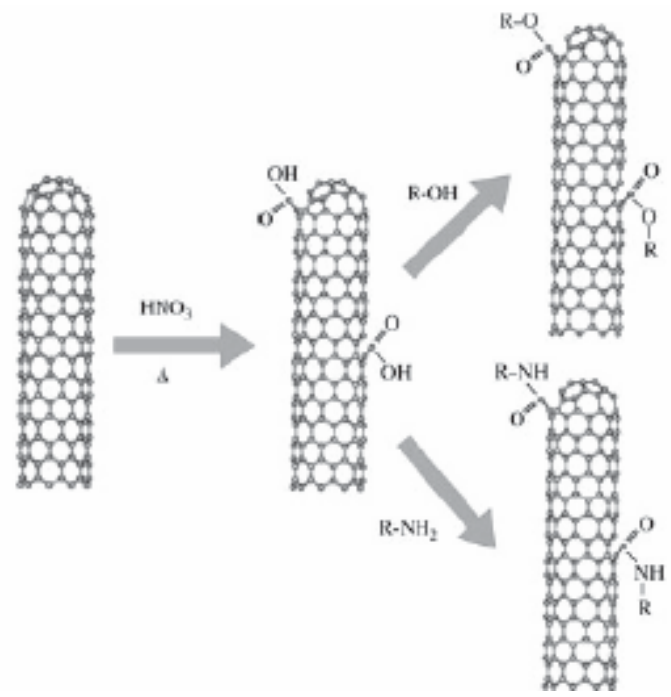

Fig. 20. Schematic representation of CNTs functionalization through oxidation followed by the esterification or amidization of the carboxyl groups 


\section{Single walled carbon nanotubes dopage}

Carbon nanotube structure derives from bidimensional structure of the graphene and has the possibility to pass from the semiconductor state with modulable gap, to metallic state by adjusting only the diameter. This is a unique property of SWCNT. A SWCNT can act as either a conductor or a semiconductor, depending on the angle of the atom arrangement along the tube. A simple method to determine if a carbon nanotube is metallic or semiconducting is to consider its indices $(n, m)$. The nanotube is metallic if $n=m$ or $n-m=3 i$, where $i$ is an integer. Otherwise, the tube is semiconducting. In general SWCNT needs to be doped for efficient used in transistor. Dopage consists on feeling in any pure or intrinsic structure generally semiconductor, impurities (atoms) to change his behavior behalf electrons. The structure gives then easily, electrons than usual and is called donor or $n$ type structure, or it receives easily electrons than usual and is called acceptor or $p$ type structure. CNTs used in transistors are often doped. SWCNTs synthesized are naturally $p$ type semiconductors, in the other hand, their induce charges are positive (holes). This phenomenon is explained by many theories. The most common is that of charge transfer from CNT to the electrode with the relative low Fermi level. Another explanation is that CNTs are doped by atoms and radical compounds present or formed during the synthesis, the gases absorption or the purification processes (Dang, 2008).

There are many ways to dope CNTs in order to obtain $\mathrm{n}$ type CNTs semiconductor. One consists on the exposure of the CNTs to electron donors like alkalis. However, the use of potassium or oxygen in CNTs dopage has induced phenomena. The easiest way of $\mathrm{n}$ type dopage is to anneale $\mathrm{p}$ type or natural synthesized CNTs.

\section{Single walled carbon nanotubes transistors}

Carbon nanotube field effect transistors utilize semiconducting single-wall CNTs to assemble electronic devices similar to MOSFETs. An individual semiconductor SWCNT is used as a channel of the transistor between the drain and the source as illustrated in Fig. 21 and Fig. 22. This has been experimentally investigated for the first time by Tans and coworkers in the year 1998 (Tans et al., 1998). Since this period, the performance of these transistors is increasing and equalizes the best silicon transistors. Then carbon nanotubes transistors seem to be among serious nanodevices promising to replace MOSFET transistors in near future. The band gap is known to be inversely proportional to the tube diameter $d$ as shown by Tomanek and Enbody (Tomanek \& Enbody, 2002) in the relation (7) bellow:

$$
E_{g}=\frac{0.9 k}{d}
$$

where $k$ is a constant. In electronic devices, it is necessary to have an important $E_{g}$ corresponding to a lower $d$ to eliminate the excessive production of charges thermal holders. However, a low $E_{g}$ gives rise to an important current.

Thus an appropriate choice of diameter is convenient. At $300 \mathrm{~K}$ temperature, standard values of bandgap are: $1.12 \mathrm{eV}$ for silicon, $\mathrm{O} .67 \mathrm{eV}$ for germanium and $1.43 \mathrm{eV}$ for GaAs. For a given $E_{g}, d$ can be determined and of course (n, m) Hamada integers (Hamada et al., 1992) according to relation (1). Two mechanisms of contact between metal (source/drain) and semiconducting SWCNT interface lead to two types of carbon nanotubes field effect transistors. 


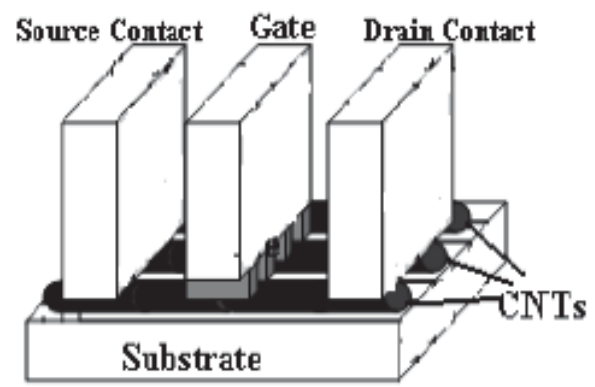

(a)

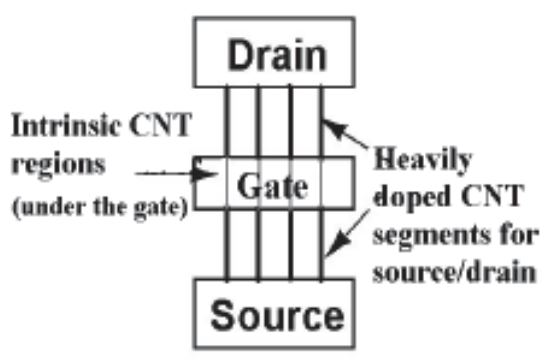

(b)

Fig. 21. Schematic diagram of carbon nanotube transistor: (b) cross section view; (a) top view

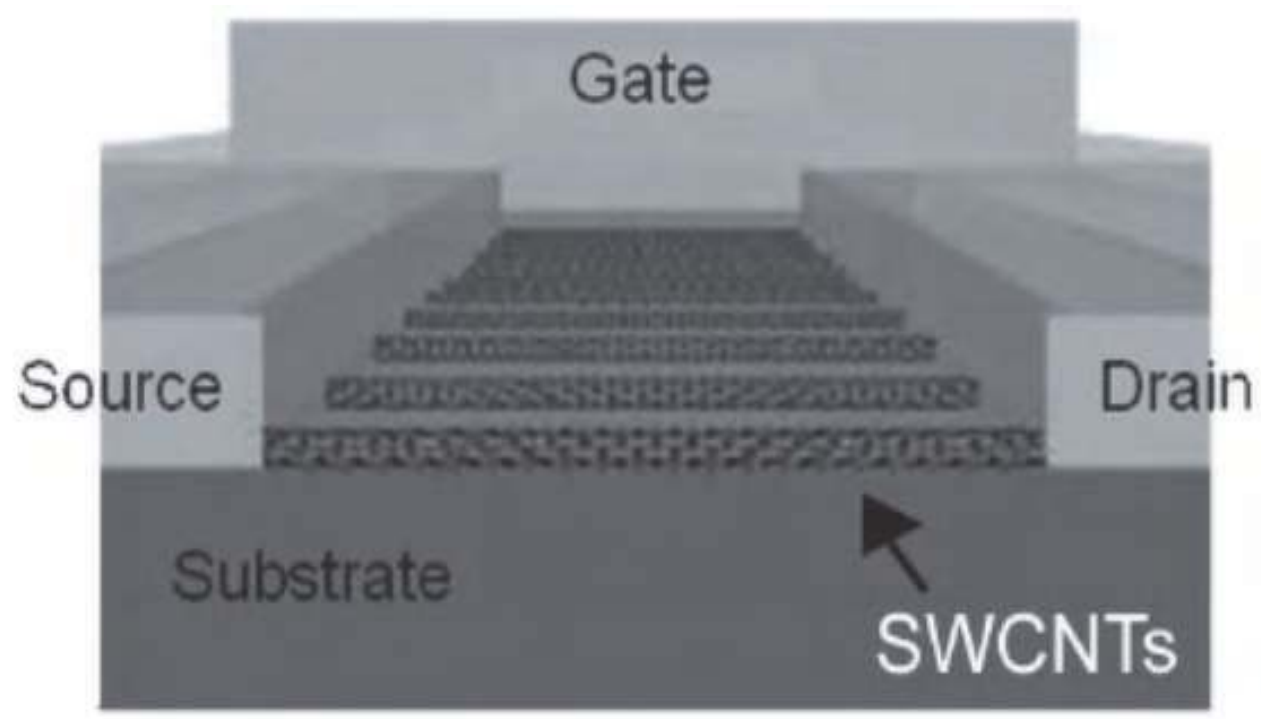

Fig. 22. The SWCNTs serve as the channels between the source and the drain in multichannel CNTFET. 
The first type is the Schottky barrier CNTFET (SB-CNTFET) or Schottky type. It is the one with the contacts operated by modulating the transmission coefficient of the Schottky barriers at the contacts or junctions between the metals (drain and source) and the SWCNT. The Schottky barrier is an energy barrier for carriers' transport, which is often a severe limitation for devices. In semiconductor devices, this problem is generally avoided by replacing metal junctions with heavily doped regions of the semiconductor. However, nanotube devices have generally relied on direct metal-semiconductor interfaces. Nonetheless, good device performance can be obtained. The reason is that the Schottky barrier represents a much less severe limitation for CNTFETs than for conventional FETs, due to the quasi-one-dimensional (1D) of the SWCNT. A carbon nanotube transistor operates as unconventional Schottky barrier transistors in which transistor action occurs primarily by varying the contact resistance rather than the channel conductance. There is not alteration of the resistivity of the channel. The Schottky barriers preclude charges to cross the junction at low gate voltage. Applying positive voltages than the gate voltage, the $\mathrm{N}$ type device suppresses the Schottky barrier and consequently increases the holes injection at the drain. The SB-CNTFET has consequently a high ambipolar characteristic.

The second type is the C-CNTFET consisting of an intrinsic SWCNT with ends doped, one $n$ type doped and another $\mathrm{p}$ doped. It is obtained by modulating the upper level of bandgap of the SWCNT. When the gate voltage is applied to the silicon back gate, the Schottky barriers are controlled or suppressed. Thus the CNTFET has an unipolar characteristic as a standard or conventional MOSFET. C-CNTFET helps to overcome the SB-CNTFET limitations like un-increasing miniaturization, which stops the enhancement of performance. According to their geometry, CNTFETs are classified into two types. The first one is the back-gate CNTFET proposed for the first time by Tans et.al. Fig. 23 presents the coaxially gated carbon nanotube transistor. This model is done to improve electrostatic coupling between the SWCNT and the gate.

\section{Intrinsic CNT}

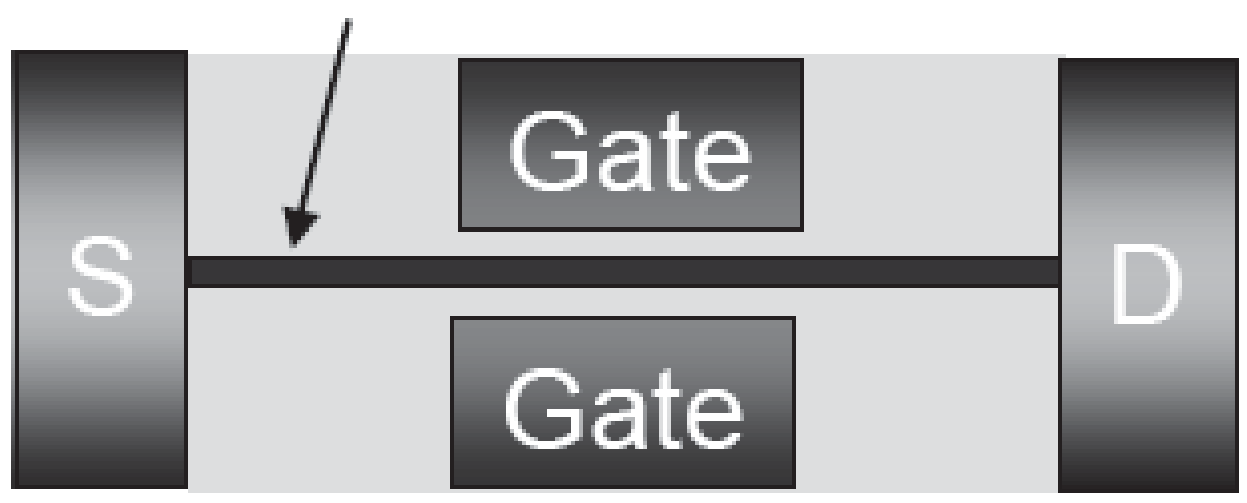

Fig. 23. Wrap-around gate CNTFET with an intrinsic SWCNT monochannel. 
In this structure a single SWCNT is used to bridge two noble metal electrodes on an oxidized silicon wafer; the SWCNT plays the role of channel and the metal electrodes act as source and drain. The heavily doped silicon wafer itself behaves as the back gate. The other one is the top gate CNTFET with gate placed over the CNT.

In contrast to conventional Si MOSFETs with source and drain contacts oppositely doped compared to the channel, CNTFETs usually have metal contacts. In interface between a metal and a SWCNT, a Schottky barrier (SB) is formed. If a metal and a semiconductor are joined and the Fermi level in the semiconductor is higher in energy than that in the metal, electrons move from the semiconductor into the metal leaving a positive background of ionized atoms. Electrons continue to be transported across the interface until the Fermi levels of the two systems have equilibrated. A SB for holes (electrons) arises due to the mismatch between the Fermi level of the metal and the valence (conduction) band of the semiconductor.

The main transport mechanisms in a metal-semiconductor contact are thermionic emission over the Schottky barriers (tunneling through the SB and recombination and generation). The barrier thickness can be reduced by increasing the electric field close to the interface by for example, increasing the doping of the semiconductor. The tunneling current is also larger in a reversed biased compared to a forward biased SB due to the thinner barrier. The measurements of the SB heights as a function of SWCNT diameter confirm that large SWCNTs diameter have to be used to obtain CNTFETs with negligible SBs. However, even though large diameter SWCNTs have the additional benefit of a high mobility (Zhou et al, 2005) their small band gaps lead to high off state currents resulting in a trade-off between switching speed and leakage current. The $\mathrm{I}_{\text {on }}$ over $\mathrm{I}_{\text {off }}$ ratio still very low compared to theoretical, this seems to be the result of the fact that SWCNTs used are a mixture of semiconductor and metallic. Towards this goal, the semiconducting tubes are now rendered non-conductive by application of an appropriate gate voltage prior to the electrochemical modification. The FETs fabricated in this manner are found to display favourable holes mobilities and a high ratio approaching 106 between the current in the ON and OFF state. However, the results show that the SB decreases with increasing SWCNT diameter, an effect which is attributed to the decrease in band gap. In addition, the measurements indicate that a SWCNT diameter larger than $2 \mathrm{~nm}$ is needed to obtain a SB height close to zero for a palladium (Pd) contacted SWCNT in vacuum. The Schottky barrier height is given by the relation (8) bellow:

$$
\Phi_{S B h}=\varphi_{S W C N T}-\varphi_{m}-\frac{E_{g}}{2}
$$

where $\varphi_{S W C N T}=5.0 \mathrm{eV}$ is the SWCNT work function,

$\varphi_{m}=5.12 \mathrm{eV}$ is the metal $(\mathrm{Pd})$ work function and $E_{g}$ gived by equation (7). The relation enhancement leads to the effective Schottky barrier height.

For many electronic applications it is of great importance to have CNTs that exhibit uniform electrical properties. The fabrication of CNTFET requires exclusively semiconducting nanotubes. At present there exists no synthesis technique that allows for a reliable control over the electrical properties of synthesized nanotubes. The raw material of SWCNTs constitutes a mixture of metallic and semiconducting single walled carbon nanotubes. 


\section{Conclusion}

Among chemical elements with impressive number of allotropes is found the carbon. These allotropes are divided in two groups: the traditionals and the carbon nanostructures. This last group is led by carbon nanotubes characterize by unique properties allowing numerous applications and have emerged as main building blocks in nanotechnology. A large part of the success of the MOSFET transistor is due to the fact that it can be scaled to increasingly smaller dimensions, which results in higher performance. Though this trend still continues, bulk MOSFET will soon reach its limiting size. For this reason, the semiconductor industry is looking for different materials and devices to integrate with the current silicon-based technology and in the long run, possibly replace it. The carbon nanotube field effect transistor is one among the most promising alternatives due to its superior electrical properties. Among the obstacles encountered in the use of CNTFET is a well-known phenomenon occurring under a vacuum environment or with a top gate oxide and top gate electrode, the undesirable ambipolarity in Schottky barrier (Martel et al, 2008). CNT-based CMOS technology is realized by suppressing the ambipolar regions of the CNTFETs with por n-type doping (Bachtold et al, 2001). Doping of CNTs with p or n-type to remove ambipolarity is still great challenge. The poor understanding of the most important factors that affect SB formation in metal-CNT interface junction stems from the difficulty in using many of the techniques available for bulk materials to perform measurements on SBs in nanoscale contacts. Carbon nanotubes are nowadays commonly used in laboratories due to their commercial availability in large quantities.

But some properties of CNTs and their applications have not yet fulfilled the expectations, while others are still object of intensive exploration. In addition, effective purification methods can ensure a reasonable quality of the samples. The recently developed chemical and electrochemical functionalization schemes have significantly extended the application spectrum of CNTs. However, the actual challenge still remains the reaching complete control in their synthesis to selectively obtain isolated SWCNTs as well as bundles, to control their location and orientation, and to synthesize complex and organized networks of arrays of CNTs directly on large substrates suitable for applications.

\section{Acknowledgements}

The author would like grateful acknowledge Mr. Abega, J. C. of the rectorate of University of Yaounde I, for his helpful advices and his logistical assistance. He thanks on the same time Dr Ben-Boli, G. H. the main responsible of the Nuclear Physics laboratory of Physics department, Faculty of Science, University of Yaounde I, Pr Mane Mane J and Dr Tiodjio Sendja, B. for many helpful discussions.

\section{References}

Bachtold, A., Hadley, P., Nakanishi, T. \& Dekker, C., Logic circuits with carbon nanotubes transistors, Science, 2001, 294, 1317

Baird, T., Fryer, J. R. \& Grant, B., Carbon formation on iron and steel foils by hydrogen pyrolysis reactions at $700^{\circ} \mathrm{C}$, Carbon $12,591,1974$ 
Baker, R. T. K., Barber, M. A., Harris, P. S., Feates, F. S. \& Waite, R. J., Nucleation and Growth of carbon deposits from the nickel catalyzed decomposition of acetylene, J. Catalysis, 26, 51, 1972

Bunch, S., Yaish, Y., Brink, M., Bolotin, K. \& McEuen, P. L., Coulomb oscillations and hall effect in quasi-2D graphite quantum dots, Nano Letters, 5, 287, 2005

Cojocaru, C S \& Le Normand, F, On the role of activation mode in the plasma and hot filament enhanced catalytic chemical vapour deposition of vertically aligned carbon nanotubes, Thin Solid Films, 515 53, 2006

Cojocaru, C. S., Synthèse contrôlée CCVD des films de nanostructures orientées de carbon, Thesis, Université Louis Pasteur, Strasbourg I, 2003

Dang, T. T., Portes logiques à base de CNTFETs -Dispersion des caractéristiques et tolérance aux défauts ; Thesis, Institut Polytechnique de Grenoble, 2008

Dresselhaus, M. S., Dresselhau,s G., Sugihara, K., Spain, I. L. \& Goldberg, H. A., Graphite Fibers and Filaments, Springer-Verlag, Berlin, 1988

Dolmatov, V. Y., Detonation synthesis ultrqdispersed diamond: properties and applications, Russ. Chem. ReV., 70, 607, 2001

Eba Medjo, R., Thiodjio Sendja, B., Mane Mane, J. \& Owono Ateba, P., A study of carbon nanotube contamination by XANES spectroscopy, Phys. Scr. 80, 045601, 2009

Eba Medjo, R., Thiodjio Sendja, B., Mane Mane, J. \& Owono Ateba, P., XAS study of the orientation of oriented carbon nanotubes films, Phys. Scr. 80, 055602, 2009

Eba Medjo, R., Structural and morphological characterization of carbon nanostructures synthesized by chemical vapour deposition using spectroscopic techniques and electron microscopies, Thesis, University of Yaounde I, 2011

Ebbesen, T. W. \& Ajayan, P.M., Large-scale synthesis of carbon nanotubes, Nature, 358, 220222,1992

Ebbesen T. W. \& Takada T., Topological and $\mathrm{sp}^{3}$ defect structures in nanotubes, Carbon, 33, 973, 1995

Hamada, N., Sawada, S. \& Oshiyama, A., "New One-Dimensional Conductors: Graphitic Microtubules", Phys. Rev. Lett. 68, 15791992

Hamon, A. L., Contribution à l'étude des nanotubes: structure et contraste en microscopie électronique, enchevètrements et comportement mécanique, Thesis, Ecole Centrale de Paris, 2001

Heimann, R. B., Kleiman, J. \& Salansky, N. M., A unified structural approach to linear carbon polytupes, Nature,306, 164, 1983

Heimann, R. B., Evsyukov, S. E. \& Kavan, L., Carbyne and Carbynoid Structures, Kluwer Academic Press, Dordrecht, 1999

Helveg, S., Lopez-Cartes, C., Sehested, J., Hansen, P., Clausen, B., Rostrop-Nielson, J., AblidPedersen, F. \& Norskov, Atomic-scale imaging of carbon nanofibre growth, Nature, 427, 426, 2004

Hirsch, A., Functionalization of single-walled carbon nanotubes, Angewandte ChemieInternational Edition 41 (11), 1853-1859, 2002

Hueczko, A., Vertically aligned carbon nanofibers and related structures, Appl. Phys. A., 74, 617, 2002

Iijima, S., Helical microtubules of graphitic carbon, Nature, 354, 56, 1991 
Ivanov, V., Nagy, J.B., Lambin, Ph., Lucas, A., Zhang, X.B., Zhang, X.F., Bernaerts ,D., Van Tendeloo, G., Amelinckx, S. \& Van Landuyt, J., Carbon nanotubes production by catalytic pyrolysis of benwene, Chem. Phys. Lett. 223, 329, 1994

Krishnan, A., Dujardin, E., Treacy, M. M. J., Hugdahl, J., Lynum, S. \& Ebbesen, T. W., Graphytic cones and the nucleation of curved carbon surfaces, Nature, 388, 451, 1997

Kroto, H. W., The stability of Fullerene Cn ( $\mathrm{n}=24,28,32,50,60$ and 70), Nature, 329, 529, 1987

Kuzmany, H., Kukovecz, A., Simona, F., Holzweber, M., Kramberger, Ch. \& Pichler, T., "Functionalization of carbon nanotubes", Synthetic Metals, 141 113-122, 2004

Kuznetsova, A., Popova, I., Yates, J. T., Bronikowski, M. J., Huffman, C. B., Liu, J., Smalley, R. E., Hwu, H. H. \& Chen, J. G. G., Oxygen-containing functional groups on singlewall carbon nanotubes: NEXAFS and vibrational spectroscopic studies, Journal of the American Chemical Society 123 (43), 10699-10704, 2001

Martel, R., Derycke, V., Lavoie, C., Appenzeller, J., Chan, K., Tersoff, J., \& Avouris, P., "Ambipolar Electrical Transport in Semiconducting Single-Wall Carbon Nanotubes," Phys. Rev. Lett. 87, 256805, 2001

Melechko, A. V., Merkulov, V. I., McKnigh,t T. E., Guillorn, M. A., Klein, K. L., Lowndes D. H. \& Simpson, M. L., Vertically aligned carbon nanofibers and related structures: Controlled synthesis and directed assembly, J. Appl. Phys., 97, 041301, 2005.

Meyyappan, M., Delzeit, L., Cassell, A. \& Hash, D., Carbon nanotube growth by PECVD: a review, Plasma Sources Science and Technology., 12, 205, 2003

Mordkovich, V. Z., Shiratori, Y., Hiraoka, H. \& Takeuchi, Y., Synthesis of multishell fullerenes by laser vaporization of composite carbon targets, Physics of the Solid State, 44, 4, 603, 2002

Novoselov, K., Geim, A. K., Morozov, S. V., Jiang, D., Zhang, Y., Dubonos, S. V., Grigorieva, I. V. \& Firsov, A.A., Electric field effect in atomically thin carbon films. Science, 306(5696), 666, 2004

Pederson, M. R. \& Broughton, J. Q., Nanocapillarity in Fullerene Tubules, Physical Review Letters, 69 (18), 2689-2692, 1992

Pierson, H. O., Handbook of carbon, graphite, diamond and fullerenes, Processing and Applications. (William Andrew Publishing/Noyes, New Jersey, 1993

Rosenberg, R. A., Love, P. J. \& Rehn, V., Polarization-dependant C(K) near-edge X-ray absorption fine structure of graphite, Phys. Rev. B 33, 4034, 1986

Sasaki S., KEK, National Laboratory for High Energy, Physics, Report, 83, 221984

Somani, P. R. \& Umeno, M., Modern Research and Educational Topics in Microscopy, ed. Mendez-VilasA \& Diaz J., Formatex Vol. 2, 634-642, 2007

Stanford University, CNFET Model website,http://nano.stanford.edu/model.php/id=23

Svensson J., Carbon Nanotube Transistors: Nanotube Growth, Contact Properties and Novel Devices, Thesis, University of Gothenburg, Göteborg, 2010

Tans, S.J., Verschueren, A.R.M. \& C. Dekker, "Room-temperature transistor based on a single carbon nanotube", Nature, Vol.393, pp.49-52, 1998

Tomanek, D. \& Enbody, R.J.; "Science and Application of Nanotubes", Fundamental Materials Research, 2002 
Zhou, X., Park, J.-Y., Huang, S., Liu, J., \& McEuen, P. L., Band structure, phonon scattering, and the performance limit of single-walled carbon nanotube transistors. Phys. Rev. Lett., 95(14), 146805, 2005. 


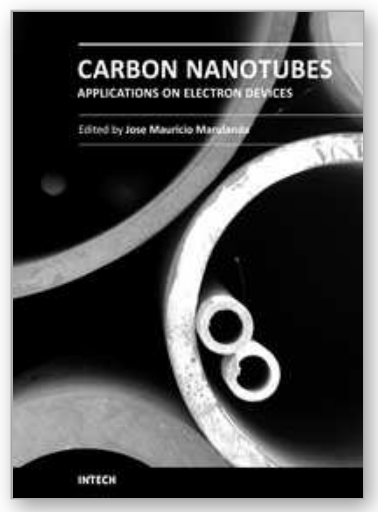

\section{Carbon Nanotubes Applications on Electron Devices}

Edited by Prof. Jose Mauricio Marulanda

ISBN 978-953-307-496-2

Hard cover, 556 pages

Publisher InTech

Published online 01, August, 2011

Published in print edition August, 2011

Carbon nanotubes (CNTs), discovered in 1991, have been a subject of intensive research for a wide range of applications. In the past decades, although carbon nanotubes have undergone massive research, considering the success of silicon, it has, nonetheless, been difficult to appreciate the potential influence of carbon nanotubes in current technology. The main objective of this book is therefore to give a wide variety of possible applications of carbon nanotubes in many industries related to electron device technology. This should allow the user to better appreciate the potential of these innovating nanometer sized materials. Readers of this book should have a good background on electron devices and semiconductor device physics as this book presents excellent results on possible device applications of carbon nanotubes. This book begins with an analysis on fabrication techniques, followed by a study on current models, and it presents a significant amount of work on different devices and applications available to current technology.

\section{How to reference}

In order to correctly reference this scholarly work, feel free to copy and paste the following:

Eba Medjo Rolant (2011). Carbon Nanotube Synthesis, Carbon Nanotubes Applications on Electron Devices, Prof. Jose Mauricio Marulanda (Ed.), ISBN: 978-953-307-496-2, InTech, Available from:

http://www.intechopen.com/books/carbon-nanotubes-applications-on-electron-devices/carbon-nanotubesynthesis

\section{INTECH}

open science | open minds

\section{InTech Europe}

University Campus STeP Ri

Slavka Krautzeka 83/A

51000 Rijeka, Croatia

Phone: +385 (51) 770447

Fax: +385 (51) 686166

www.intechopen.com

\section{InTech China}

Unit 405, Office Block, Hotel Equatorial Shanghai

No.65, Yan An Road (West), Shanghai, 200040, China 中国上海市延安西路65号上海国际贵都大饭店办公楼 405 单元

Phone: +86-21-62489820

Fax: $+86-21-62489821$ 
(C) 2011 The Author(s). Licensee IntechOpen. This chapter is distributed under the terms of the Creative Commons Attribution-NonCommercialShareAlike-3.0 License, which permits use, distribution and reproduction for non-commercial purposes, provided the original is properly cited and derivative works building on this content are distributed under the same license. 